\title{
Exponentially Handsome Proof Nets and Their Normalization
}

\author{
Matteo Acclavio \\ Department of Computer Science \\ University of Luxembourg \\ matteoacclavio.com
}

\begin{abstract}
Handsome proof nets were introduced by Retoré as a syntax for multiplicative linear logic. These proof nets are defined by means of cographs (graphs representing formulas) equipped with a vertices partition satisfying simple topological conditions. In this paper we extend this syntax to multiplicative linear logic with units and exponentials. For this purpose we develop a new sound and complete sequent system for the logic, enforcing a stronger notion of proof equivalence with respect to the one usually considered in the literature. We then define combinatorial proofs, a graphical proof system able to capture syntactically the proof equivalence, for the cut-free fragment of the calculus. We conclude the paper by defining the exponentially handsome proof nets as combinatorial proofs with cuts and defining an internal normalization procedure for this syntax.
\end{abstract}

\section{Introduction}

One of the novelties introduced by linear logic [15] was the syntax of proof nets. Proof nets are a graphical syntax [26, 27] for proofs able to capture the proof equivalence in the multiplicative fragment of linear logic (denoted MLL): proof nets are canonical representative of equivalent proofs modulo independent rules permutations. In addition, proof nets are a sound and complete proof system in the sense of [9] for MLL, since it is possible to check if a graph represents a correct derivation in polynomial time with respect to the size of the graph. This test can be conducted by means of a topological criterion, often refereed to as correctness criterion [15, 12, 18, 44].

Several extensions of proof nets have been proposed to cover multiplicative linear logic with units $\left(M L L_{u}\right)$, but none of them can be considered to be fully satisfactory. In presence of the units, the correctness criterion requires to add additional edges, called jumps, to a proof net in order to connect the gates of the unit $\perp$ to an axiom or an unit 1 [22].

The quest for a satisfactory syntax for $\mathrm{MLL}_{u}$-proofs has come to an end after the publication of [21] where is shown that it is not possible to have at the same time a syntax capturing the whole MLL $_{u}$ proof equivalence and a polynomial correctness criterion, unless $\mathrm{P}=\mathrm{NP}$. This result depends on the presence of the jumps: on one hand they are needed in order to check in polynomial time if the proof net is correct, but on the other hand they enforce a coarse notion of proof equivalence which requires to "rewire" the jumps to capture the full proof equivalence.

A similar problem occurs in the multiplicative exponential linear logic (MELL) due to the presence the weakening rule ${ }^{1}[51,50]$. Moreover, the presence of the promotion rule in this fragment poses an additional difficulty since this rule is context-sensitive. However this latter problem is easily addressed by including in the proof net syntax the so called boxes whose scope is to delimit portions of the graph [35, 30, 32, 3, 2], as shown in Figure 1 .

Handsome proof nets are an alternative syntax for MLL proofs introduced by Retoré in [39, 44] using the results from his $\mathrm{PhD}$ thesis [37]. In this syntax, the information contained in a proof is represented

\footnotetext{
${ }^{1}$ Indeed, the decidability of MELL is still an open question and depends on the presence of the weakening rule [25, 49].

U. Dal Lago and V. de Paiva (Eds.) Second Joint International Workshop on Linearity \& Trends in Linear Logic and Applications. EPTCS 353, 2021, pp. 1-25 doi 10.4204/EPTCS.353.1

(C) Matteo Acclavio

This work is licensed under the Creative Commons Attribution License.
} 

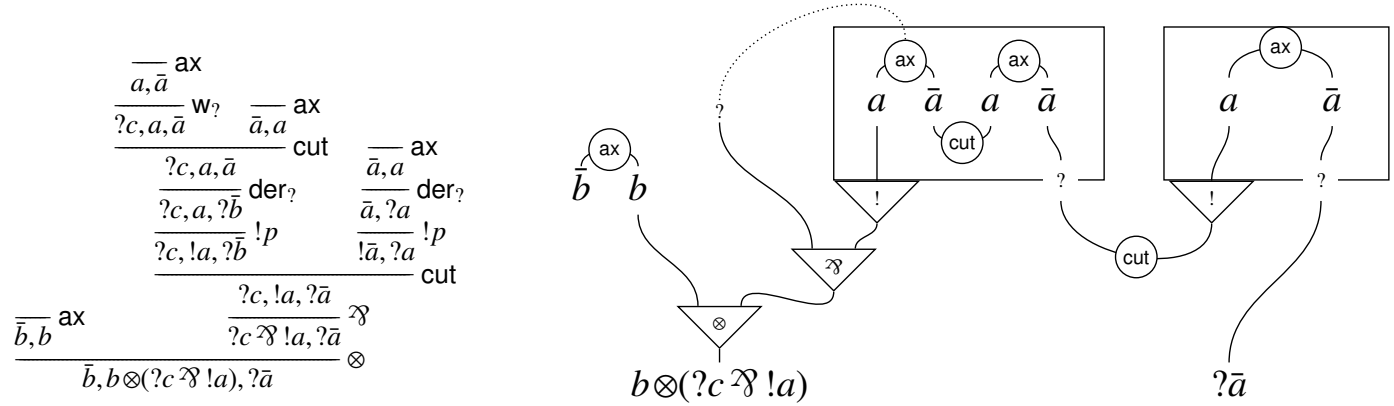

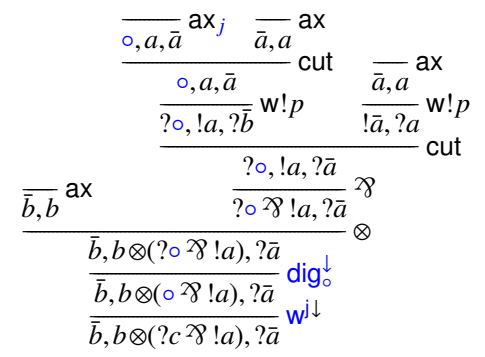

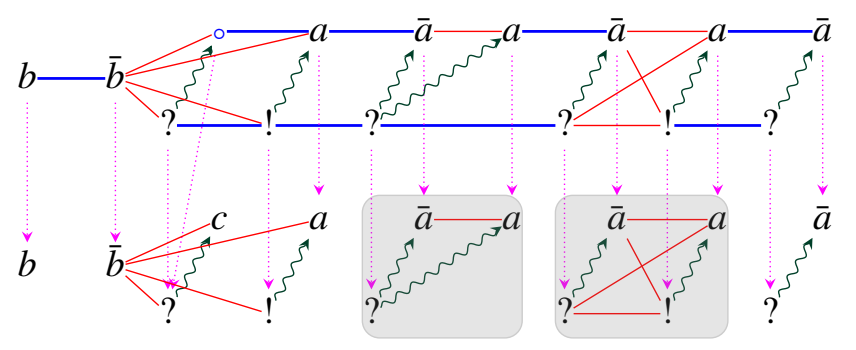

Figure 1: Upper row: a derivation of the sequent $\bar{d}, d \otimes(? c$ ช $! a), ?(\bar{a} \otimes \bar{b}), ? b$ in MELL and a corresponding proof net (with jump drawn as a dotted edge). Lower row: a decomposed derivation in MELL $L^{j}$ of the same sequent and its corresponding exponentially handsome proof net. In this latter, the gray shadings represent cut-rules, and the vertex $\circ$ represents the jump.

by specific graphs encoding formulas (called cographs ${ }^{2}$ ) together with a perfect matching which satisfies specific topological conditions. For this reason, we this syntax is called RB-cographs.

Combinatorial proofs were introduced by Hughes [23, 24, 46] to capture proof equivalence in classical logic [47, 4]. They can be considered as an extension of handsome proof nets since they are defined as specific graph homomorphisms from a RB-cograph to a cograph encoding a formula. In particular, combinatorial proofs indirectly give us a decomposition result, allowing to separate the "linear" part of the proof, that is, the part containing the logical interactions between its components, from the "resource management" part, that is, the part taking care of erasing and duplicating components.

Contribution of the paper. In this paper we extend Retoré's handsome proof nets for MLL and we define a syntax able to represent proofs in $\mathrm{MLL}_{u}$ and MELL. For this purpose, we define combinatorial proofs for cut-free MELL-derivations, and then we show how to also encode derivation with cuts.

To achieve our goal, we first definite of a new sound and complete proof system for MELL, called MELL ${ }^{j}$, and we prove cut-elimination for it. This system contains the weak promotion rule from light linear logic [17, 28, 31] and the digging rule instead of regular promotion. Moreover, the system uniquely assigns each unit $\perp$ and each weakening rule to an branch of a derivation thanks to ad-hoc rules. This choice allows us to mimic jumps assignation in proof nets and reduce the complexity of proof equivalence.

We then extend the results in [6] in order to define combinatorial proofs for MELL ${ }^{j}$. This allows us to represent equivalent cut-free derivations in MELL'. We show that this syntax has a polynomial correctness criterion and is able to syntactically capture the proof equivalence, that is, equivalent MELL ${ }^{j}$-derivations are represented by the same syntactic object.

We conclude by defining exponentially handsome proof nets as extensions of the combinatorial

\footnotetext{
${ }^{2} \mathrm{~A}$ cograph is a graph containing no induced subgraph isomorph to the four-vertices path $\mathrm{P}_{4}$. In [13] it is shown that a graph encodes a formula iff it is a cograph.
} 


$$
\underset{a, \bar{a}}{\operatorname{ax}} \frac{\Gamma, A, B}{\Gamma, A \ngtr B} \text { \& } \frac{\Gamma, A \quad B, \Delta}{\Gamma, A \otimes B, \Delta} \otimes \frac{\Gamma}{\Gamma, \perp} \perp-1 \frac{A, ? \Gamma}{! A, ? \Gamma} ! p \frac{\Gamma, A}{\Gamma, ? A} \operatorname{der}_{?} \frac{\Gamma}{\Gamma, ? A} \mathrm{w}_{?} \frac{\Gamma, ? A, ? A}{\Gamma, ? A} \mathrm{c}_{?} \mid \frac{\Gamma, A \quad \bar{A}, \Delta}{\Gamma, \Delta} \text { cut }
$$

Figure 2: Sequent calculus rules for MELL and the cut-rule

proofs syntax. Exponentially handsome proof nets allow us to encode derivations containing cuts, hence to compose proofs. Finally, we provide a cut-elimination procedure for this syntax by means of a terminating graph rewriting.

Organization of the paper. In Section 2 we discuss the notion of proof equivalence for the standard MELL sequent calculus. Then we define a sound and complete proof system MELL for MELL, where MELL-proof equivalence is restricted. We then prove a decomposition theorem for MELL $L^{j}$ using deep inference rules [19, 20, 8]. In Sections 3 to 5 we define the three components needed to define combinatorial proofs for MELL'. In particular, in Section 3 we recall relation webs [19, 7], which generalize cographs, and we show how they can be used to encode formulas with modalities. In Section 4 we extend the correctness criterion for Retoré's RB-cographs to relation webs with special matchings, called RGB-cographs, which encode the linear part of a MELL' proof. In Section 5 we define the MELLfibrations taking care of encoding the resource management part of our proofs. In Section 6 we define combinatorial proofs as MELL-fibrations from an RGB-cograph to a relation web. Finally, in Section 7 . we define exponentially handsome proof nets as MELL-combinatorial proofs of sequents containing additional formulas keeping track of the cut-rules, and we provide a cut-elimination procedure for this syntax.

\section{Proof Systems for MELL}

In this section we recall the sequent systems for multiplicative exponential linear logic (with units) and its subsystems. We then discuss in Section 2.2 the notion of proof equivalence for these logics and in Section 2.3 we define a sound and complete proof system for MELL, enforcing a coarser notion of proof equivalence. In Section 2.4 we show that this new proof system admits a decomposition theorem, which we exploit in Section 6 to define the syntax of combinatorial proofs for MELL.

We define formulas in negation normal form generated from a countable set of propositional variables $\mathcal{A}=\{a, b, \ldots\}$ and set of constants $\{1, \perp, \circ\}^{3}$ by the following grammar:

$$
A, B::=a|\bar{a}| A \not 8 B|A \otimes B| ! A|? A| \perp|1| \circ
$$

A literal is a formula of the shape $a$ or $\bar{a}$ for an $a \in \mathcal{A}$. A MELL-formula is a formula containing no occurrences of $\circ$. Linear negation ${ }^{-}$is defined on MELL formulas through the De Morgan laws: $\overline{\bar{A}}=A$, $\overline{A \otimes B}=\bar{A}$ ×8 $\bar{B}, \overline{! A}=? \bar{A}, \overline{1}=\perp$. A sequent $\Gamma=A_{1}, \ldots, A_{n}$ is a non-empty multiset of formulas.

In this paper we consider multiplicative linear logic and its extensions with units and and exponentials $4^{4}$ denoted respectively by MLL, MLL ${ }_{u}$ and MELL [15]. We use the same names to denote the cut-free sequent calculi for these logics defined using the sequent calculus rules in Figure 2.

$$
\operatorname{MLL}=\{a x, \otimes, \not\}\} \quad \operatorname{MLL}_{u}=\operatorname{MLL} \cup\{\perp, 1\} \quad \operatorname{MELL}=\operatorname{MLL}_{\mathrm{u}} \cup\left\{! p, \mathrm{w}_{?}, \mathrm{c}_{?}, \mathrm{der}_{?}\right\}
$$

We say that a formula $F$ is provable in $\mathrm{X}$ (denoted by $\stackrel{\mathrm{X}}{\longleftarrow} F$ ) if there is a derivation of $F$ in the system X.

\footnotetext{
${ }^{3}$ The symbols $\perp$ and 1 are called units. The symbol $\circ$ is a special symbol which we use as a "placeholder" for $\perp$ and weakening rules.

${ }^{4}$ In this paper, where not otherwise specified, we consider multiplicative exponential linear logic including units.
} 


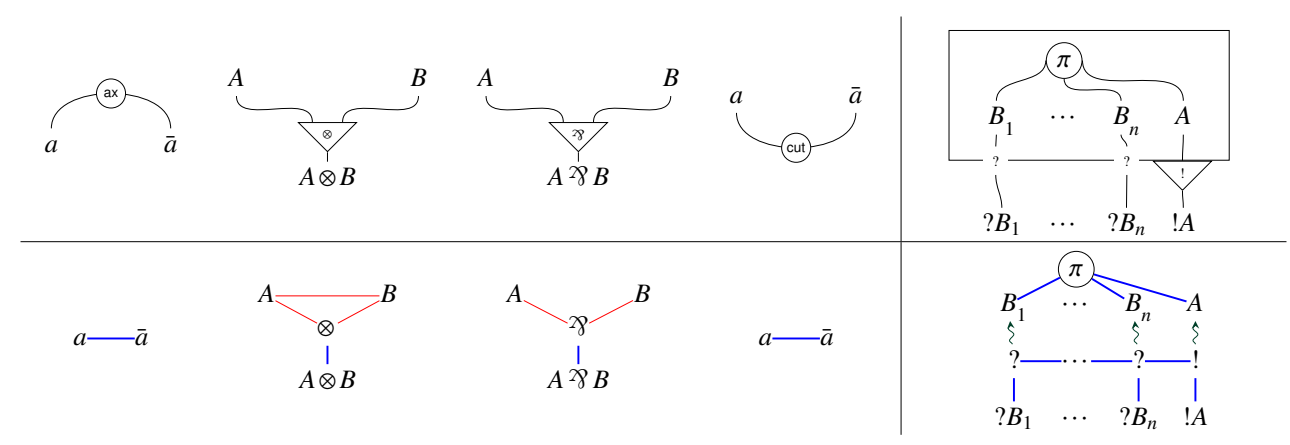

Figure 3: Upper row: gates for MLL proof nets and the way the promotion rule is encoded. Lower row: gates for RB-proof nets and the intuition on how the promotion rule should be encoded in this syntax.

We recall the cut-elimination result for MELL, which encompasses also MLL and $M L L_{u}$.

Theorem 1 ([15]). Let $F$ be a formula. Then $F$ is provable in MELL iff it is provable in MELL $\cup$ \{cut\}.

\subsection{From Proof Nets to Handsome Proof Nets}

Proof nets are a graphical syntax for proofs in linear logic introduced by Girard in [15]. In this syntax, MLL proofs are represented by replacing each instance of a rule by a corresponding gate whose inputs are the active formulas of the rule and whose outputs are the principal formulas, as shown in the upper row of Figure 3 . The graphs generated by these gates are called proof structures and some of them do not have a corresponding proof in MLL. Therefore, a topological correctness criterion needs to be defined to decide whether a proof structure is a proof net, i.e., is the translation of a MLL proof. Beside Girard's duable trip condition, several alternative criteria have been proposed in the literature [12, 18].

Using RB-graphs, which are graphs with two kind of edges (Red or Regular, and Blue or Bold), Retoré defined in [39] the syntax of RB-proof nets where gates are represented as the RB-graphs, as shown in the lower row of Figure 3 . In this syntax the correctness criterion can be formulated by requiring the absence of elementary (i.e., non self-intersecting) cycles made of alternating colours edges. From RB-proof nets, he then discovered the syntax of handsome proof nets (or RB-cographs) by using the transformation in Equation (11) below, allowing to remove from an RB-graph all the nodes which are not labelled by literals [39, 40, 44]. This syntax is formally presented in Section 4. Aim of this paper is to further develop RB-cographs by adding an encoding of the linear logic modalities and units.

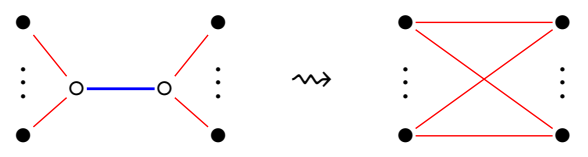

where the labels of the nodes $\circ$ are not literals

The encodings for modalities we employ in this paper uses the same kind of directed edges from the handsome proof nets for pomset logic [38, 7, 41, 42, 44, 43] (we here represent these edges by green squiggly arrows instead of red arrows). The upper row of the rightmost column of Figure 3 shows the usual encoding of the promotion rule of MELL by means of boxes isolating a portion of the proof net. In the lower row of the same column we provide the intuitive representation of how the same box should be represented in terms of the RB-proof nets. This intuition might help the reader familiar with proof nets; however, it is not further developed in the paper, as our approach focuses on generalizing RB-cographs. 


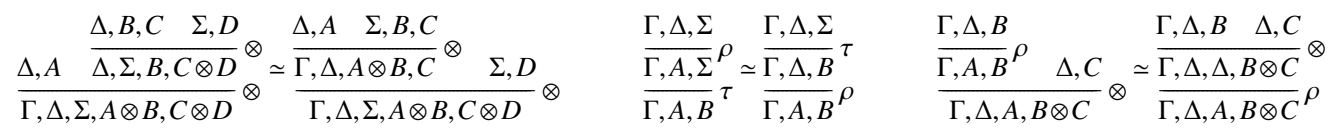

Figure 4: Independent rules permutations defined for all $\rho, \tau \in\left\{\ngtr,, \perp, \mathbf{w}_{?}, \mathbf{C}_{?}, \operatorname{der}_{?}\right\}$.

$$
\begin{aligned}
& \frac{\Gamma, ? A_{1}, ? A_{2}, ? A_{3}}{\Gamma, ? A_{1}, ? A} \frac{\Gamma, ? A_{1}, ? A_{2}, ? A_{3}}{\Gamma, ? A} \mathrm{c}_{?} \simeq \frac{\frac{\Gamma, ? A, ? A}{\Gamma, ? A, ? A_{3}}}{\Gamma, ? A} \mathrm{c}_{?} \quad \frac{\frac{\Gamma, ? A}{\Gamma, ? A} \mathrm{c}_{?}}{\Gamma, ? A, ? A} \mathrm{w}_{?} \simeq \Gamma, ? A, ? A \quad \mathrm{w}_{?} \simeq \Gamma, ? A
\end{aligned}
$$

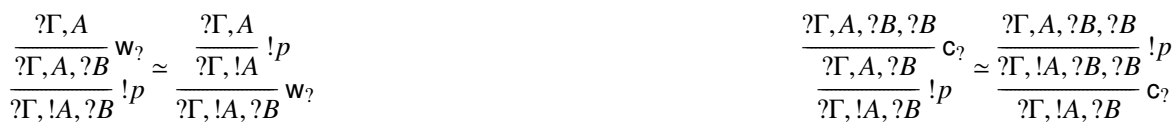

Figure 5: Additional rules permutations. The first line is called the weakening-contraction comonad

\subsection{Proof Equivalence}

The notion of proof equivalence in MELL is defined (e.g., in [36, 11]) as the equivalence relation $\simeq$ over MELL-derivations generated by independent rules permutations, i.e., the permutations of rules which have disjoint sets of principal and active formulas shown in Figure 4, together with the ones in Figure 5. It is worth noticing that these rules permutations are heavily used in the proof of cut-elimination theorems.

As shown in [21], the instances of rule permutations in the last line of Figure 4 involving $\perp$-rule are responsible of the $\mathrm{P}$-space complexity bound of checking proof equivalence in $M L L_{u}$. In fact, these rules permutations allows to move the $\perp$ formula between different derivation branchings as shown in the following example, where the leftmost and rightmost derivations, which are equivalent, are "naturally" represented by two different proof nets.

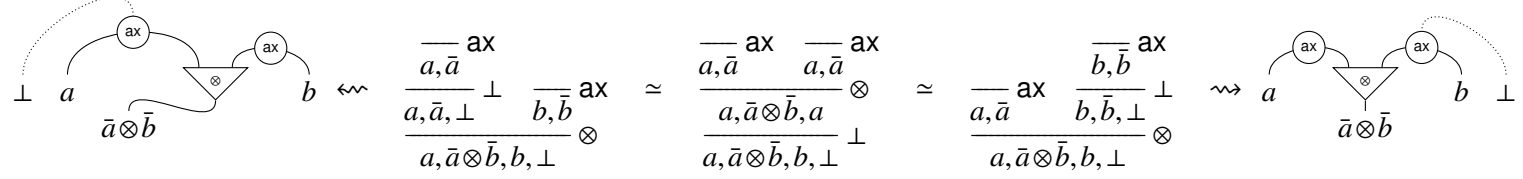

As consequence of this complexity result, we cannot design a syntax $\mathcal{S}$ for $\mathrm{MLL}_{\mathrm{u}}$ satisfying the two following desiderata at the same time under the assumption $P \neq N P$ :

- correctedness in $\mathcal{S}$ can be checked in polynomial time: we can check in polynomial time if an object expressed in the syntax $\mathcal{S}$ represents a correct proof in $\mathrm{MLL}_{\mathrm{u}}$;

- $\mathcal{S}$ captures proof equivalence: if $\llbracket \pi \rrbracket$ and $\llbracket \pi^{\prime} \rrbracket$ are the encodings in $\mathcal{S}$ of two derivations $\pi$ and $\pi^{\prime}$ in MELL such that $\pi \simeq \pi^{\prime}$, then $\llbracket \pi \rrbracket=\llbracket \pi^{\prime} \rrbracket$.

The same argument applies to MELL-derivations in presence of the rule $\mathbf{w}_{\text {? }}$. The complexity of checking proof equivalence depends on the fact that each $\mathbf{w}_{\text {? }}$ and $\perp$ must be assigned to an instance of ax or of 1 by permuting them upwards in a derivation. We refer to such assignation as jump. Since $\simeq$ allows to re-assign jumps, the equivalence check has to test all possible jumps, whose number is exponential with respect to the number of $\perp$ and $w_{\text {? }}$ in a derivation.

\subsection{Restricting Proof Equivalence in MELL}

As consequence of [21], we cannot aspire to design a proof system 5 which captures the proof equivalence $\simeq$ of MELL. To overcome this problem, in this subsection we define MELL ${ }^{j}$, a sound and complete proof

\footnotetext{
${ }^{5}$ In the sense of [9], that is, in which we can check if a syntactic object is correct in polynomial time.
} 


$$
\overline{a, \bar{a}, \circ_{1}, \ldots, \circ_{n}} \operatorname{ax}_{j}^{n} \quad{\overline{1, \circ_{1}, \ldots, \circ_{n}}}_{j}^{n} \quad \frac{\Gamma, \circ}{\Gamma, \perp} \perp^{j} \quad \frac{\Gamma, \circ}{\Gamma, ? A} \mathrm{w}^{\mathrm{j}} \mid \frac{A, \Gamma}{! A, ? \Gamma} \mathrm{w} ! p \quad \frac{? ? A, \Gamma}{? A, \Gamma} \operatorname{dig}_{?} \frac{\Gamma, ? \circ}{\Gamma, \circ} \operatorname{dig}_{\circ}
$$

Figure 6: On the left: the sequent rules fixing jump assignations. On the right: the weak promotion rule, the digging rule and the additional digging rule for $\circ$

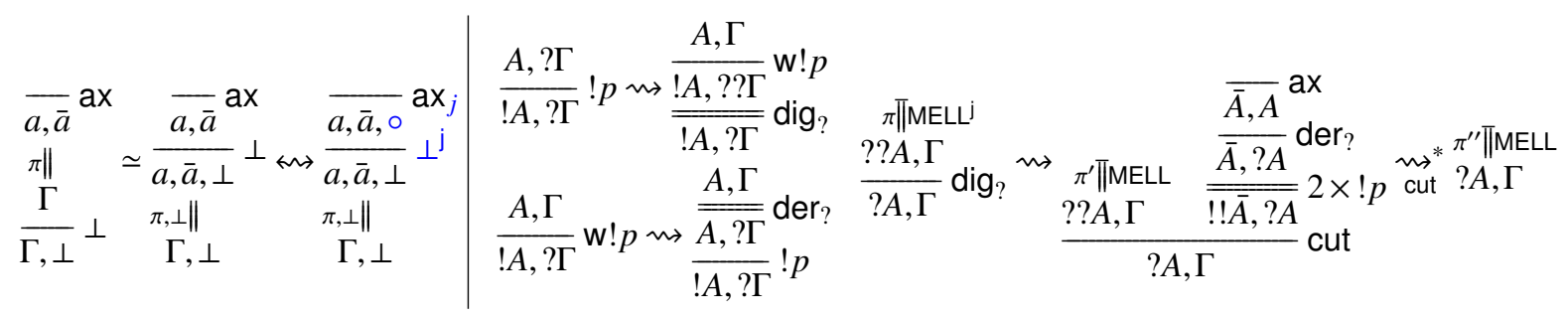

Figure 7: How to transform a $\perp$ associate to a specific ax-rule to a $\perp^{\mathrm{j}}$ associate to a $a x_{j}$-rule, and how to replace $! p$ with a derivation using $\mathrm{w} ! p$ and $\operatorname{dig}_{\text {? }}$ and vice versa. Note that elimination of the $\operatorname{dig}_{\text {? }}$ introduces a cut, which can be eliminated relying on the cut-elimination result in MELL.

system for MELL enforcing a coarser proof equivalence, denoted by $\simeq$, with respect to $\simeq$. In fact, in MELL ${ }^{j}$ each $\perp$ - and $w_{\text {? }}$-rule instance is uniquely associated to a specific ax- or 1-rule instance, mimicking the way in which the corresponding nodes are linked by jumps in a proof net.

We define the following sequent systems using the rules in Figures 2 and 6

$$
\operatorname{MLL}_{\mathrm{u}}^{\mathrm{j}}=\left\{\mathrm{ax}_{j}, 1_{j}, \perp^{\mathrm{j}}, \ngtr, \otimes\right\} \quad \mathrm{MELL}^{\mathrm{j}}=\left\{\mathrm{ax}_{j}, 1_{j}, \perp^{\mathrm{j}}, \mathrm{w}^{\mathrm{j}}, \not>, \otimes, \mathrm{w} ! p, \operatorname{der}_{?}, \operatorname{dig}_{?}, \operatorname{dig}_{\circ}, \mathrm{C}_{?}\right\}
$$

where $\operatorname{ax}_{j}=\left\{\operatorname{ax}_{j}^{n} \mid n \in \mathbb{N}\right\}$ and $1_{j}=\left\{1_{j}^{n} \mid n \in \mathbb{N}\right\}$. The proof equivalence $\simeq$ over MELL $\mathcal{L}^{j}$ derivations is defined as in Figure 4 by considering $\rho$ and $\tau$ ranging over $\left\{\perp^{j}, w^{j},{ }^{\prime}, \operatorname{der}_{?}, \operatorname{dig}_{?}, \operatorname{dig}_{\circ}, \mathrm{c}_{?}\right\}$ plus the associativity of contraction, that is, the rule permutation in the left-hand side of Figure 5 .

In these systems the relation between one $\perp^{j}$ - or $w^{j}$-rule and one $a_{j}$ - or $1_{j}$-rule is syntactically encoded in the sequent system syntax, as jumps in proof nets are. Each instance of $a x_{j}$ and $1_{j}$ introduces a bunch of jump place-holders denoted by $\circ$. Since each occurrence of a $\circ$ is unique, each place-holder is further used by a single $\perp$ or $\mathrm{w}_{\text {? }}$ instance.

Remark 2. Another solution to uniquely associate $\perp$ and $w_{\text {? }}$ to an axiom would be to introduce an axiom rule with non-empty contexts, i.e., a rule having as conclusion any sequent of the shape $\Gamma, a, \bar{a}$, as done in sequent calculus for classical logic $\mathrm{G}$ [52]. Such axiom rule would keep track of whole information of the weakened formula, making the syntax of the structures described in Section 4 heavier.

Moreover, in MELL ${ }^{\mathrm{j}}$ we replace the promotion rule ! $p$ with the weak promotion rule $\mathrm{w} ! p$ used in light linear logic [17] and in soft linear logic [28, 31] and the digging rule. This choice is motivated by the fact that weak promotion allows to group the ! introduced by a promotion with all the ? of its context formulas. In particular, weak promotion is a context-free rule, that is, it can be applied independently form the shape of the premise context, while the regular promotion rule $! p$ is a context-sensitive rule, in the sense that it can be applied only if the context is of the form ?Г. The digging rules $\operatorname{dig}_{\text {? }}$ and $_{\text {dig }}$ are required to make the system sound and complete with respect to MELL.

Theorem 3. If $F$ is a fomula, then $\stackrel{\mathrm{x}}{\longrightarrow} \mathrm{F}$ iff $\stackrel{\mathrm{X}^{j}}{\longmapsto} F$.

Proof. By rules permutations, we can move each occurrence $\rho$ of a $\mathbf{w}_{\text {? }}$ - or a $\perp$-rule up in the derivation until it reaches the assigned occurrence $\sigma_{\rho}$ of an $\mathrm{ax}_{j}$ - or a $1_{j}$-rule. Then we replace $\sigma_{\rho}$ with an occurrence 


$$
\begin{aligned}
& \frac{\overline{a, \bar{a}, \circ, \cdots, \circ}}{a, \bar{a}, \circ, \cdots, \circ, \circ^{\prime}, \cdots, \circ^{\prime}} \mathrm{ax}_{j} \overline{a, \bar{a}, \circ^{\prime}, \cdots, \circ^{\prime}} \mathrm{ax}_{j} \rightsquigarrow_{\mathrm{cut}} \frac{}{a, \bar{a}, \circ, \cdots, \circ, \circ^{\prime}, \cdots, \circ^{\prime}} \mathrm{ax}_{j}
\end{aligned}
$$

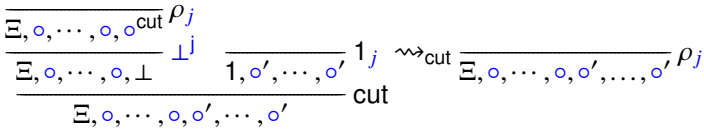

$$
\begin{aligned}
& \frac{\frac{\Gamma, A, B}{\Gamma, A \ngtr B} \times \frac{\Delta, \bar{A} \quad \Sigma, \bar{B}}{\Delta, \Sigma, \bar{A} \otimes \bar{B}} \otimes{ }_{\Gamma, \Delta, \Sigma} \varlimsup_{\mathrm{cut}} \frac{\Gamma, A, B \quad \Delta, \bar{A}}{\Gamma, \Delta, B} \text { cut } \quad \Sigma, \bar{B}}{\Gamma, \Delta, \Sigma} \text { cut } \\
& \frac{\frac{\Gamma, A}{? \Gamma, ! A} \mathrm{w} ! p \quad \frac{\bar{A}, \Delta, B}{? \bar{A}, ? \Delta, ! B} \mathrm{w} ! p}{? \Gamma, ? \Delta, ! B} \text { cut } \rightsquigarrow_{\mathrm{cut}} \frac{\Gamma, A \bar{A}, \Delta, B}{\frac{\Gamma, \Delta, B}{? \Gamma, ? \Delta, ! B} \mathrm{cut}} \mathrm{w} p
\end{aligned}
$$

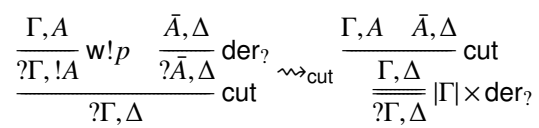

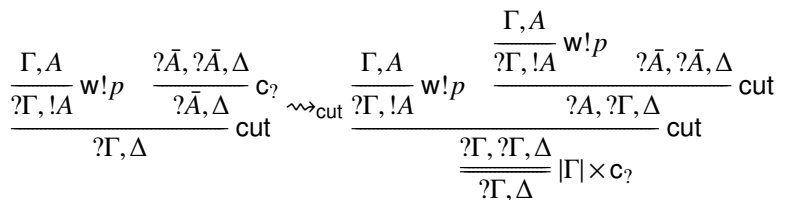

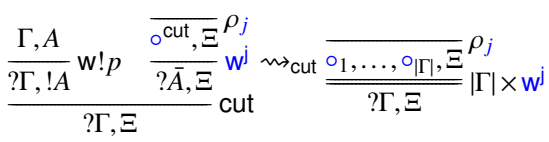

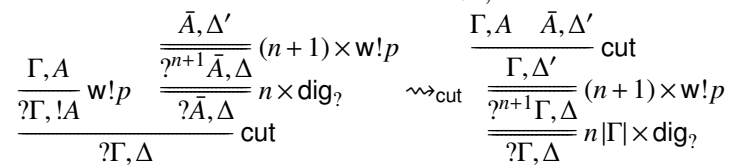

Figure 8: The cut-elimination steps in MELL' ${ }^{\mathrm{j}}$, where $\rho_{j} \in\left\{\mathrm{ax}_{j}, 1_{j}\right\}$ is the unique rule introducing the $\circ^{\text {cut }}$ which is the active premise of the $\perp^{j}$ or $w^{j}$ involved in the cut-elimination step.

of the same rule $\sigma_{\rho}$ with an additional $\circ$ in the conclusion, and $\rho$ with an occurrence of $\rho^{\mathrm{j}}$ applied to this fresh $\circ$ (an example is shown in Figure 7). Moreover, every ! $p$ can be replaced by a $w ! p$ followed by a finite number of dig $_{\text {? }}$ and vice versa by MELL cut-elimination theorem [15] (see Figure 7).

Strictly speaking, the proof system MELL does not satisfy the subformula property because of the presence of the rules $\mathrm{dig}_{\text {? }}$ and dig $_{\circ}$. However, we can prove a cut-elimination result. Observe that a weaker notion of the subformula property holds since all formulas that can appear in a derivation of an arbitrary sequent are going to be subformulas of a formula at the root or formulas of the form ? $\cdots ? A$ where $? A$ is a subformula of a formula at the root. At the moment this paper is written, the decidability of MELL is an open question [49]. Thus we do not focus on proof search for MELL'.

Theorem 4. Let $F$ be a formula. Then $F$ is provable in $\mathrm{MELL}^{\mathrm{j}}$ iff $F$ is provable in $M E L L^{\mathrm{j}} \cup\{$ cut\}.

Proof. Assuming the equivalences $\simeq \mathrm{J}$, we define the rewritings in Figure 8, which decrease the size of the cut-formula, and the ones in Figure 9, which permute the rule $w ! p$ above $d e r_{?}, \operatorname{dig}_{?}, \operatorname{dig}_{\circ}, w^{j}, \perp^{j}$ and $\mathrm{c}_{\text {? }}$, permute the rules der? dig $_{\text {? }}$ and $\operatorname{dig}_{\circ}$ above $\mathrm{w}^{j}, \perp^{j}$ and $\mathrm{c}_{\text {? }}$, and permute the rules $\mathrm{w}^{\mathrm{j}}$ and $\perp^{j}$ above $\mathrm{c}_{\text {? }}$.

Note that the cut-elimination steps involving $\circ$ are non-local rewritings which reassign or introduce new $\circ$ in $\mathrm{ax}_{j}$ or $1_{j}$ rules. Moreover, after the rules in Figure 9, the step involving dig ? has to involve at least two different $\mathrm{w} ! p$.

To prove cut-elimination we define some rewriting steps behaving similarly to the ones used in [1], that is, where rewriting deals with multiple boxes at a time.

\subsection{Decomposing MELL Proofs}

In order to prove the decomposition result for our system MELL', we introduce deep inference rules [19. 20, 8]. These rules can be applied at any depth of the sequent, i.e., to any subformula occurring in it, allowing us to push to the bottom of the derivation the $\mathrm{w}_{?}, \mathrm{c}_{\text {? }}$, dig ? and der $_{\text {? }}$ inferences.

We denote by $\Gamma\{\}$ a context, which is a sequent or a formula with an "hole" in place of a formula, and we define the following sets of rules, composed of sequent rules from Figure 2 and the deep inference 


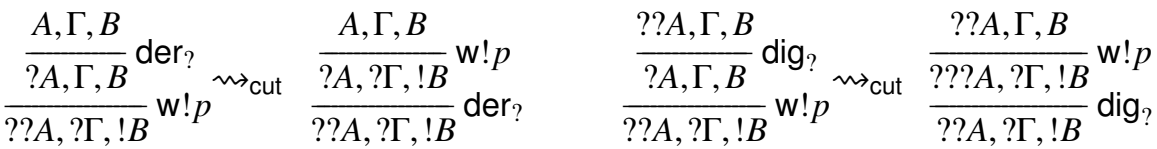

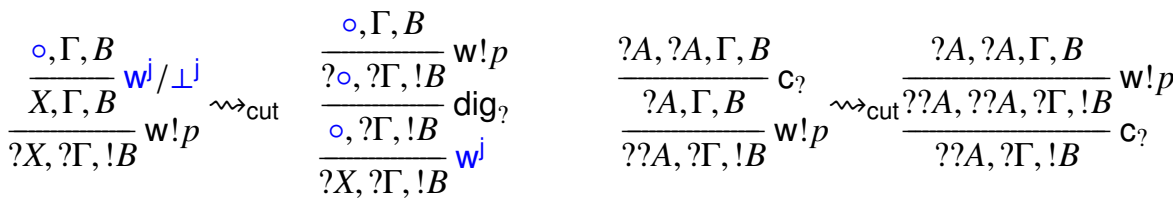

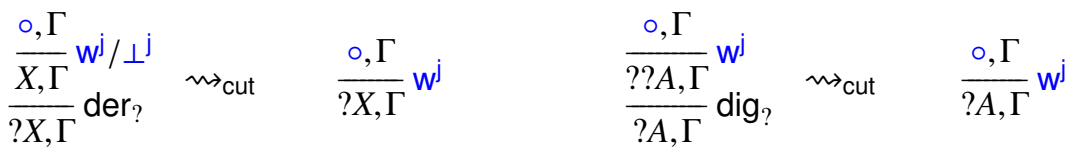

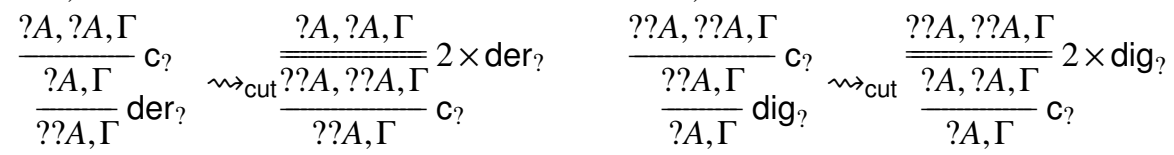

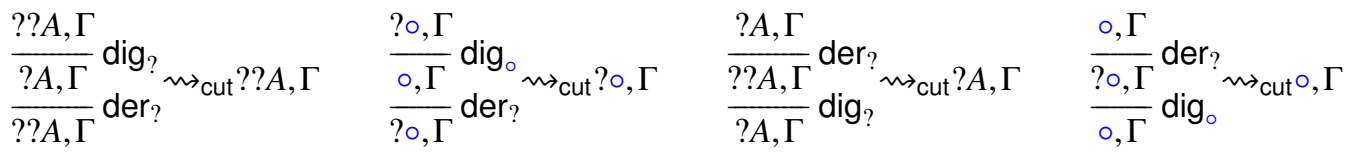

Figure 9: Commutative cut-elimination steps

$$
\frac{\Gamma\{A\}}{\Gamma\{? A\}} \operatorname{der}_{?}^{\downarrow} \frac{\Gamma\{? ? A\}}{\Gamma\{? A\}} \operatorname{dig}_{?}^{\downarrow} \frac{\Gamma\{? \circ\}}{\Gamma\{\circ\}} \operatorname{dig}_{\circ}^{\downarrow}\left|\frac{\Gamma\{\circ\}}{\Gamma\{\perp\}} \perp \frac{\Gamma\{\circ\}}{\Gamma\{? A\}} \mathrm{w}_{?}^{\downarrow}\right| \frac{\Gamma\{? A \ngtr ? A\}}{\Gamma\{? A\}} \mathrm{c}_{?}^{\downarrow}
$$

Figure 10: Deep inference rules for dereliction and digging, for $\perp$ and ?-weakening, and for ?contraction.

rules from Figure 10 .

$$
\begin{aligned}
& \mathrm{MLL}^{\ell}=\mathrm{MLL} \quad \mathrm{MLL}_{\mathrm{u}}^{\ell}=\left\{\mathrm{ax}_{j}, 1_{j}, \not 8, \otimes\right\} \quad \mathrm{MELL}^{\ell}=\left\{\mathrm{ax}_{j}, 1_{j}, \not \varnothing, \otimes, \mathrm{w} ! p\right\} \\
& \mathrm{MLL}^{\downarrow}=\emptyset \quad \mathrm{MLL}_{\mathrm{u}}^{\downarrow}=\left\{\perp^{\downarrow}\right\} \quad \mathrm{MELL}^{\downarrow}=\left\{\operatorname{der}_{?}^{\downarrow}, \operatorname{dig}_{?}^{\downarrow}, \operatorname{dig}_{\circ}^{\downarrow}, \perp^{\downarrow}, \mathrm{w}_{?}^{\downarrow}, \mathrm{c}_{?}^{\downarrow}\right\}
\end{aligned}
$$

Theorem 5. Let $F$ be a formula. Then $\stackrel{\mathrm{MELL}}{\longleftarrow} F$ iff there is a formula $F^{\prime}$ such that $\stackrel{\mathrm{MELL}}{\ell} F^{\prime} \stackrel{\mathrm{MELL}}{\longmapsto} F$. More precisely, if $\stackrel{\mathrm{MELL}}{\longmapsto} F$, then there are some formulas $F^{\prime}, F^{\prime \prime}$ and $F^{\prime \prime \prime}$ such that

$$
\stackrel{\mathrm{MELL}}{\ell}^{\ell} F^{\prime} \stackrel{\left\{\mathrm{der}_{?}^{\downarrow}, \mathrm{dig}_{?}^{\downarrow}, \mathrm{dig}_{0}^{\downarrow}\right\}}{\longmapsto} F^{\prime \prime} \stackrel{\left\{\mathrm{w}_{?}^{\downarrow}, \perp \downarrow\right.}{\longmapsto} F^{\prime \prime \prime} \stackrel{\left\{\mathrm{c}_{?}^{\downarrow}\right\}}{\longmapsto} F
$$

Proof. After Proposition 3 , it suffices to replace each occurrence of $\operatorname{dig}_{?}$, dig, der $_{\text {? }}, \perp^{j}, w^{j}$, and $c_{\text {? }}$ by an occurrence of their corresponding deep version $\operatorname{dig}_{?}^{\downarrow}$, dig $g_{\circ}^{\downarrow}$, der? $\perp^{\downarrow}, w_{?}^{\downarrow}$, and $c_{?}^{\downarrow}$ and then to push these inferences to the bottom of the derivation 6 .

The converse is proven by reverting the previous argument, that is, by pushing up all deep rules applications and replace them by the corresponding non-deep rules.

\footnotetext{
${ }^{6} \mathrm{~A}$ part of these rules permutations may be performed without using deep inference rules, as shown in Figure 9 , but they are not enough to prove the result.
} 


\section{Relation Webs}

Cographs are graphs encoding formulas constructed using a conjunction and a disjunction connective [13]. In this section we present modal relation webs, which generalize cographs, and which will be used in the next sections to encode MELL-formulas We define modal relation webs as mixed graphs (i.e., graphs with both directed and undirected edges) satisfying certain topological conditions. Moreover, we show that they identify formulas modulo associativity and commutativity of $\otimes$ and $\not 2$.

A directed graph $\mathcal{G}=\left\langle V_{\mathcal{G}}, \stackrel{\mathcal{G}}{\rightsquigarrow}\right\rangle$ is a set $V_{\mathcal{G}}$ of vertices equipped with an irreflexive binary edge rela-

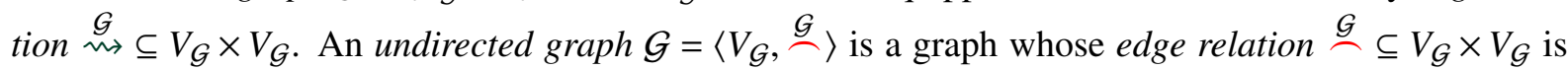
irreflexive and symmetric. A mixed graph is a triple $\mathcal{G}=\left\langle V_{\mathcal{G}}, \stackrel{\mathcal{G}}{\stackrel{\mathcal{G}}{\rightsquigarrow}\rangle}\right\rangle$ where $\left\langle V_{\mathcal{G}}, \stackrel{\mathcal{G}}{\stackrel{G}{ }}\right\rangle$ is an undirected graph and $\left\langle V_{\mathcal{G}}, \stackrel{\mathcal{G}}{\rightsquigarrow}\right\rangle$ is a directed graph, such that $\stackrel{\mathcal{G}}{\sim} \stackrel{\mathcal{G}}{\rightsquigarrow}=\emptyset$ and $\stackrel{\mathcal{G}}{\rightsquigarrow}$ is irreflexive. We omit the index/superscript $\mathcal{G}$ when it is clear from the context. When drawing a graph we draw $v-w$ whenever $v \frown w$, and $v \leadsto w$ whenever $v \rightsquigarrow w$; otherwise we either draw no edge at all, or we draw $v \cdots w$ when we want to underline the absence of edges. A mixed graph is $\mathcal{L}$-labeled if each vertex $v$ carries a label $l(v)$ selected from a label set $\mathcal{L}$. In this paper we fix the label set to be $\mathcal{L}=\mathcal{A} \cup \overline{\mathcal{A}} \cup\{!, ?, 1, \perp, \circ\}$.

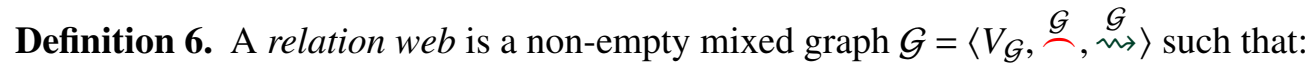

- $\stackrel{\mathcal{G}}{\rightsquigarrow}$ is transitive and irreflexive;

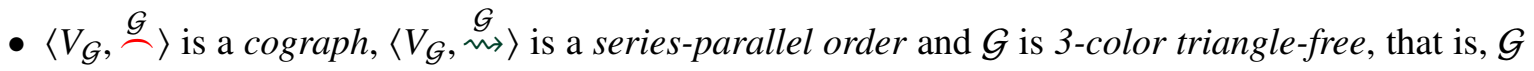
contains no induced subgraphs of the following shape:

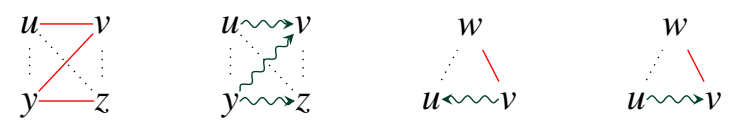

A cograph is an undirected graph containing no induced subgraph as the leftmost one in Equation (3).

Let $\mathcal{G}$ and $\mathcal{H}$ be two disjoint mixed graphs. We define the following operations:

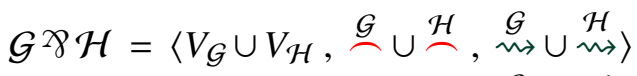

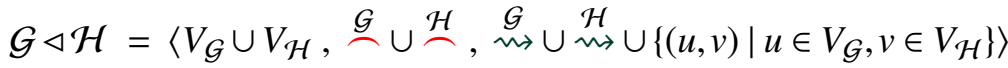

$$
\begin{aligned}
& \mathcal{G} \otimes \mathcal{H}=\left\langle V_{\mathcal{G}} \cup V_{\mathcal{H}}, \stackrel{\mathcal{G}}{\sim} \cup \stackrel{\mathcal{H}}{\sim} \cup\left\{(u, v),(v, u) \mid u \in V_{\mathcal{G}}, v \in V_{\mathcal{H}}\right\}, \stackrel{\mathcal{G}}{\cdots} \cup \stackrel{\mathcal{H}}{\rightsquigarrow}\right\rangle
\end{aligned}
$$

which can be visualized as follows:
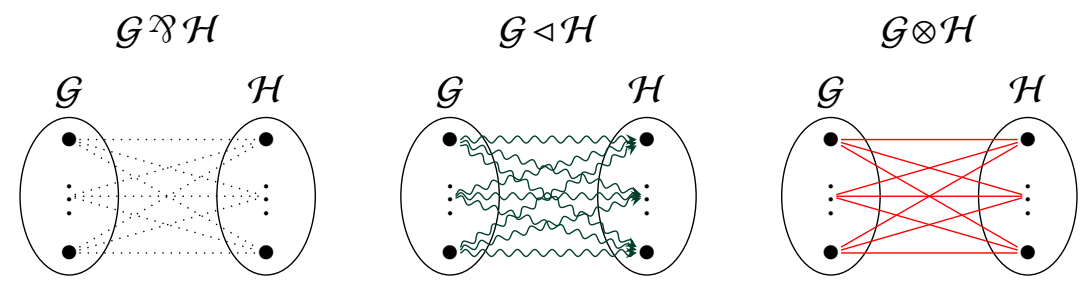

Theorem 7 ([19, 7]). A mixed graph is a relation web if and only if it can be constructed from single vertices using the three operations $\not 8, \triangleleft$ and $\otimes$ defined in Equation (4). 
For each formula $F$ we define the $\mathcal{L}$-labeled relation web $\llbracket F \rrbracket$. We use the notations $a, \bar{a}, !, ?, 1, \perp$ and - for the graph consisting of a single vertex that is labeled with $a, \bar{a}, !, ?, 1, \perp$ and $\circ$ respectively.

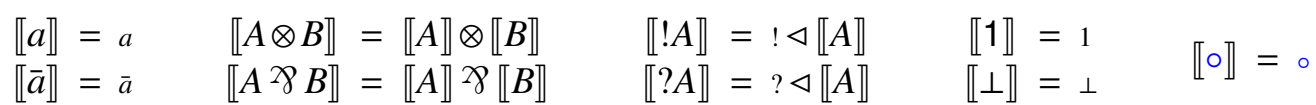

For a sequent $\Gamma=A_{1}, \ldots, A_{n}$ we define $\llbracket \Gamma \rrbracket=\llbracket A_{1}, \ldots, A_{n} \rrbracket=\llbracket A_{1} \rrbracket \not 8 \ldots \not 8 \llbracket A_{n} \rrbracket$.

Definition 8. A relation web $\mathcal{G}$ is properly labeled if for each $v, w \in V_{\mathcal{G}}$ we have $v \rightsquigarrow w$ iff $l(v) \in\{!, ?\}$.

Moreover, we say that a relation web $\mathcal{G}$ is modal if it is properly labeled and for any vertices $u, v$, $w$ with $u \rightsquigarrow w$ and $v \rightsquigarrow w$ we have $u \rightsquigarrow v$ or $v \rightsquigarrow u$ or $u=v$, i.e., $\mathcal{G}$ does not contain the two configurations below.

$$
\text { Forbidden configurations for modal relation webs: } u^{3} s^{2} v
$$

By adapting the proofs in [6], we have the following results:

Theorem 9. A relation web is the translation of a formula iff it is modal.

Proof. If $\mathcal{G}=\llbracket F \rrbracket$ for some formula $F$, then each vertex with an outgoing $\rightsquigarrow$-edge is the encoding of the modality of a subformula of $F$, hence such vertex is labeled with! or ?. Moreover, if two distinct such vertices $u$ and $v$ have a $\rightsquigarrow$-edge to some vertex $w$, then that $w$ is the encoding of a modality or an atom occurring in a subformula in the scope of the modalities corresponding to $u$ and $v$. Thus one of such modalities is in the scope of the other and we have $u \rightsquigarrow v$ or $v \rightsquigarrow u$. The converse follows from Theorem 7 and the fact that the operation $\triangleleft$ in Equation (4) is associative.

If $\mathcal{G}$ is a modal relation web, we denote by $V_{\mathcal{G}}^{\bullet}, V_{\mathcal{G}}^{1}, V_{\mathcal{G}}^{\circ}, V_{\mathcal{G}}^{!}$and $V_{\mathcal{G}}^{?}$ the set of vertices in $V_{\mathcal{G}}$ with labels respectively in $\mathcal{A} \cup \overline{\mathcal{A}},\{1\},\{\circ\},\{!\}$ and $\{?\}$. We call atomic, unit, jump, and modal vertices the ones respectively in $V_{\mathcal{G}}^{\bullet}, V_{\mathcal{G}}^{1}, V_{\mathcal{G}}^{\circ}$, and $V_{\mathcal{G}}^{! ?}=V_{\mathcal{G}}^{!} \cup V_{\mathcal{G}}^{?}$.

Proposition 10. Given a set $V_{\mathcal{G}}$ and two binary edge relations $\stackrel{\mathcal{G}}{\stackrel{G}{W}}$ and $\stackrel{\mathcal{G}}{\rightsquigarrow}$ on vertices it can be checked

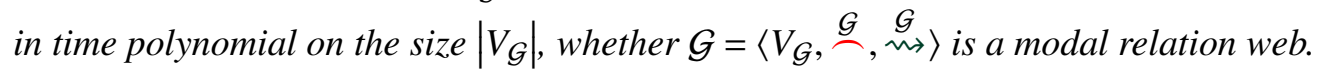

Proof. Checking the transitivity and irreflexivity of $\stackrel{\mathcal{G}}{\rightarrow}$ and symmetry of $\stackrel{\mathcal{G}}{\sim}$ is polynomial on $\left|V_{\mathcal{G}}\right|$. Then, to check the absence of the forbidden configurations in (3) and (6) we just check all triples and quadruples of vertices, which is $\mathbf{O}\left(\left|V_{\mathcal{G}}\right|^{4}\right)$. Checking the property of being properly labeled is liner on $\left|V_{\mathcal{G}}\right|$.

By associativity of the graph operations $\not 2, \otimes$ and $\triangleleft$ in Equation (4) and the commutativity of $\not 8$ and $\otimes$ we have the following equivalence.

Proposition 11. For two formulas $F$ and $F^{\prime}$, we have $\llbracket F \rrbracket=\llbracket F^{\prime} \rrbracket$ iff $F$ and $F^{\prime}$ are equivalent modulo associativity of and commutativity of $\otimes$ and $\not$.

\section{RGB-Cographs}

As discussed in Section 2.1. RB-cograph are an alternative syntax for MLL proof nets. In this paper we consider RGB-cographs from [6], which are an extension of the RB-cograph syntax. We provide a further extension of RGB-cographs and we establish a correspondence between these graphs and $\mathrm{MELL}^{\ell}$-proofs. 


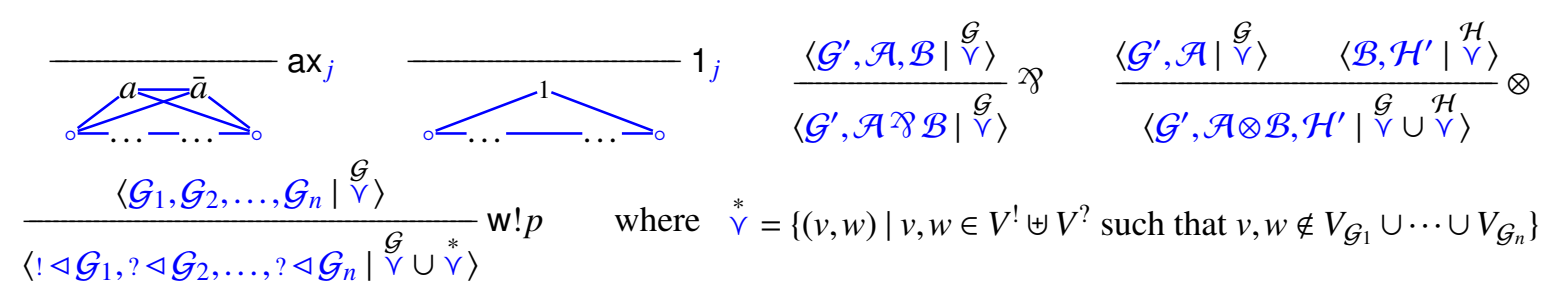

Figure 11: Translating MELL ${ }^{\ell}$ sequent proofs into RGB-cographs

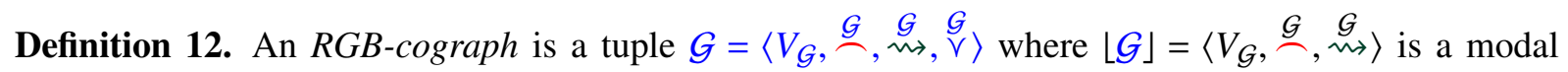
relation web, and $\stackrel{\mathcal{G}}{\vee}$ is an equivalence relation over $V_{\mathcal{G}}$, called the linking, such that

- if $v \in V_{\mathcal{G}}^{\bullet}$ then there is exactly another $w \in V_{\mathcal{G}}^{\bullet}$ with $v \vee w$ and $v \neq w$;

- if $v \in V_{\mathcal{G}}^{1}$ then $w \in V_{\mathcal{G}}^{\circ}$ for all $w \vee v$ such that $w \neq v$;

- if $v \in V_{\mathcal{G}}^{\circ}$ then there is a $u \in V_{\mathcal{G}}^{\bullet} \cup V_{\mathcal{G}}^{1}$ such that $w \vee v$;

- if $v \in V_{\mathcal{G}}^{!} \cup V_{\mathcal{G}}^{?}$ then there is a unique $w \in V_{\mathcal{G}}^{!}$such that $w \vee v$ and no $w \in V_{\mathcal{G}}^{\circ}$ such that $w \vee v$;

In particular, an $R B$-cograph is an RGB-cograph $\mathcal{G}$ with $V_{\mathcal{G}}=V_{\mathcal{G}}^{\bullet}$.

These conditions can be interpreted as follows: the jumps vertices are associated to either to a pair of atomic vertices or to a single unit vertex; modal vertices are grouped in classes containing a unique vertex in $V_{\mathcal{G}}^{!}$. For readers familiar with proof nets syntax, o-vertices can be seen as placeholders for the proof net jumps while the $\vee$-classes containing ! and ?-vertices can be thought as encoding borders of boxes, where the unique !-vertex is the box principal ports and the ?-vertices are the auxiliary ports. More precisely, the content of the box delimited by a $\vee$-class is the induced suggraph containing all the vertices $v$ such that $w \leadsto v$ for a $w$ in the $\vee$-clas 7

In drawing an RGB-cograph we use bold (blue) edges $v-w$ when $v \neq w$ and $v \vee w$. We may omit edges which can be deduced by transitivity:

Definition 13. An $x$-path in an RGB-cograph $\mathcal{G}$ is an elementary path $x_{0}, x_{1}, \ldots, x_{n}$ in the graph $\langle V, \frown U$ $\rightsquigarrow \cup \vee>$ whose edges are alternating in $\vee$ and in $\rightsquigarrow \cup \frown$. A chord in an æ-path is an edge $x_{i} \frown x_{j}$ or $x_{i} \leadsto x_{j}$ for $i, j \in\{0, \ldots, n\}$ and $i+2 \leq j$. A chordless $a$-path is an æ-path without chord. An $a$-cycle is an æ-path such that $x_{0}=x_{n}$. An RGB-cograph $\mathcal{G}$ is $a$-connected if any two vertices are connected by a chordless $æ$-path, and $\mathcal{G}$ is $a$-acyclic if it contains no chordless $\mathfrak{x}$-cycle.

Connectedness and acyclicity are used to define the following notions of correctness.

Definition 14. We say that an RGB-cograph $\mathcal{G}$ is MELL-correct if it satisfies the following conditions:

1. $V_{\mathcal{G}} \neq \emptyset$ and $\mathcal{G}$ is $æ$-connected and $æ$-acyclic;

2. if $w \stackrel{\mathcal{G}}{\sim} v$ and $v \vee v^{\prime}$, then there is $w^{\prime} \vee w$ such that $w^{\prime} \stackrel{\mathcal{G}}{\rightsquigarrow} v^{\prime}$.

We say that RGB-cograph is MLL-correct (or $\mathrm{MLL}_{\mathrm{u}}$-correct) if MELL-correct and $V=V^{\bullet}$ (respectively $\left.V=V^{\bullet} \cup V^{\circ}\right)^{8}$

Lemma 15. Let $\mathrm{X} \in\left\{\mathrm{MLL}, \mathrm{MLL}_{\mathrm{u}}, \mathrm{MELL}\right\}$ and $F$ be a formula. If $\frac{\mathrm{X}^{\ell}}{F}$ then there is a $\mathrm{X}$-correct $R G B$ $\operatorname{cograph} \mathcal{G}$ such that $\lfloor\mathcal{G}\rfloor=\llbracket F \rrbracket$.

\footnotetext{
${ }^{7} \mathrm{~A}$ similar process of reconstructing boxes from the paths in the graph can be found in [29].

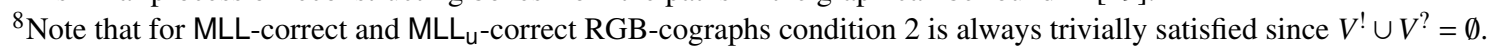


Proof. Let $\pi$ be a derivation of $F$ in X. We define a derivation of a RGB-cograph $\mathcal{G}$ such that $\lfloor\mathcal{G}\rfloor=\llbracket F \rrbracket$ by induction on the size of $\pi$ using the rules in Figure 11. In fact, all these rules preserve the condition in Definition 14.

Lemma 16. Let $\mathrm{X} \in\left\{\mathrm{MLL}, \mathrm{MLL}_{\mathrm{u}}, \mathrm{MELL}\right\}$ and $\mathcal{G}$ be a $R G B$-cograph with $\lfloor\mathcal{G}\rfloor=\llbracket F \rrbracket$. If $\mathcal{G}$ is $\mathrm{X}$-correct, then $\stackrel{\mathrm{X}^{\ell}}{\longmapsto} F$.

Proof. If $\mathrm{X}=\mathrm{MLL}$, then each MLL-correct RGB-cograph is an æ-connected æ-acyclic RB-cograph, the result is proven in [37, 40, 44]. The proof proceeds by induction on the size of an RB-cograph $\mathcal{G}$ showing that each RB-cograph is either an $\vee$-class or there is a splitting, that is, $V_{\mathcal{G}}=U \uplus V$ and if $u \stackrel{\mathcal{G}}{\vee}$ then either $u, v \in U$ or $u, v \in V$, and there are $U^{\prime} \subset U$ and $V^{\prime} \subset V$ such that for all $u \in U$ and $v \in V, u \stackrel{G}{\sim} v$ iff $u \in U^{\prime}$ and $u \in V^{\prime}$. In particular, each $\vee$-class $\{a, \bar{a}\}$ of a MLL-correct RGB-cograph $\mathcal{G}$ encodes an ax-rule with conclusion $a, \bar{a}$, and each splitting encodes a $\otimes$-rule.

If $X=M L L_{u}$, then the statement straightforwardly follows the the previous result. In this case each $\checkmark$-class encodes either an $a x_{j}$-rule if it contains a pair of atomic vertices, or a $1_{j}$-rule in case it contains a unit vertex.

If $\mathrm{X}=\mathrm{MELL}$, the proof strategy is to define for each MELL-correct RGB-cograph $\mathcal{G}$ a MLL $\mathrm{M}_{\mathrm{u}}$-correct RGB-cograph $\partial(\mathcal{G})$, and then apply the previous results. Then we shall use the derivation corresponding to $\partial(\mathcal{G})$ to reconstruct a derivation for $\mathcal{G}$.

Definition of $\partial(\mathcal{G})$ : We define $\partial(\mathcal{G})$ as follows. We define a vertex set $V^{*}=\left\{v^{\prime}, \bar{v}^{\prime} \mid v \in V_{\mathcal{G}}^{!} \uplus V_{\mathcal{G}}^{?}\right\}$ and let $V_{\partial(\mathcal{G})}=V_{\mathcal{G}}^{\bullet} \uplus V_{\mathcal{G}}^{1} \uplus V_{\mathcal{G}}^{\circ} \uplus V^{*}$, i.e., we replace in $\mathcal{G}$ each modal vertex by a dual pair of atomic vertices, that are linked by $\stackrel{\mathcal{G}}{\vee}$. Moreover, the relation $\stackrel{\partial(\mathcal{G})}{\vee}$ is the same as in $\stackrel{\mathcal{G}}{\vee}$ on vertices in $V_{\mathcal{G}}^{\bullet} \uplus V_{\mathcal{G}}^{1} \uplus V_{\mathcal{G}}^{\circ}$. In order to define $\stackrel{\mathcal{G}}{\mathrm{s}}$, we define the following relation:

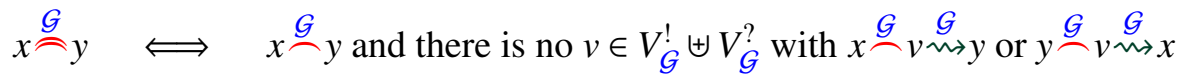

Now, let $x \stackrel{\partial(\mathcal{G})}{\stackrel{y}{y}}$ iff one of the following cases holds:

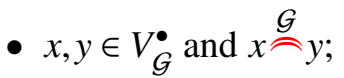

- $x \in V_{\mathcal{G}}^{\bullet}$ and $y=w^{\prime}$ for some $w \in V_{\mathcal{G}}^{!} \uplus V_{\mathcal{G}}^{?}$ with $x \stackrel{\mathcal{G}}{\sim} w$;

- $x=v^{\prime}$ and $y=w^{\prime}$ for some $v, w \in V_{\mathcal{G}}^{!} \uplus V_{\mathcal{G}}^{?}$ with $v \stackrel{\mathcal{G}}{\curvearrowright} w$;

- $x=\bar{v}^{\prime}$ for some $v \in V_{\mathcal{G}}^{!} \uplus V_{\mathcal{G}}^{?}$ and $y \in V_{\mathcal{G}}^{\bullet}$ with $v \stackrel{\mathcal{G}}{\sim} y$;

- $x=\bar{v}^{\prime}$ and $y=w^{\prime}$ for some $v, w \in V_{\mathcal{G}}^{!} \uplus V_{\mathcal{G}}^{?}$ with $v \stackrel{\mathcal{G}}{\rightsquigarrow} w$;

- $x=\bar{v}^{\prime}$ for some $v \in V_{\mathcal{G}}^{!} \uplus V_{\mathcal{G}}^{?}$ and $y \in V_{\mathcal{G}}^{\bullet}$ and there is a $u \in V_{\mathcal{G}}^{!} \uplus V_{\mathcal{G}}^{?}$ with $v \stackrel{\mathcal{G}}{v} u \stackrel{\mathcal{G}}{\sim} y$;

- $x=\bar{v}^{\prime}$ and $y=w^{\prime}$ for some $v, w \in V_{\mathcal{G}}^{!} \uplus V_{\mathcal{G}}^{?}$ and there is a $u \in V_{\mathcal{G}}^{!} \uplus V_{\mathcal{G}}^{?}$ with $v \stackrel{\mathcal{G}}{\vee} u \stackrel{\mathcal{G}}{\rightsquigarrow} w$;

Properties of $\partial(\mathcal{G})$. The intuition behind this construction can be explained using Theorem 7 . Following [19], we use the term BV-formula for an expression built from the atoms and the symbols !, ? and $\circ$ using the binary operations $\not \varnothing, \otimes$, and $\triangleleft$. In [19] it is shown that BV-formulas, modulo associativity 
and commutativity of $\ngtr$ and $\otimes$, and associativity of $\triangleleft$, are in one-to-one correspondence with relation webs via (4) and Theorem 11. We write $\mathrm{fm}(\mathcal{G})$ and $\partial(\mathrm{fm}(\mathcal{G}))=\mathrm{fm}(\partial(\mathcal{G}))$ for a corresponding BV-formula expression for respectively $\mathcal{G}$ and $\partial(\mathcal{G})$. If $v_{1}, \ldots, v_{n} \in V_{\mathcal{G}}^{!} \uplus V_{\mathcal{G}}^{?}$ form an $\stackrel{\mathcal{G}}{\vee}$-equivalence class, this means that $\mathrm{fm}(\mathcal{G})$ is of shape $F\left\{v_{1} \triangleleft B_{1}\right\} \cdots\left\{v_{n} \triangleleft B_{n}\right\}$ for some $n$-ary context $F\{\} \cdots\{\}$ (because $\mathcal{G}$ is modal). We can reformulate the translation above as follows:

$$
\partial\left(F\left\{v_{1} \triangleleft B_{1}\right\} \cdots\left\{v_{n} \triangleleft B_{n}\right\}\right)=\left(\bar{v}_{1}^{\prime} \otimes \cdots \otimes \bar{v}_{n}^{\prime} \otimes \partial\left(B_{1} \not \supset \cdots \curlyvee B_{n}\right)\right) \ngtr \partial \partial\left(F\left\{v_{1}^{\prime}\right\} \cdots\left\{v_{n}^{\prime}\right\}\right)
$$

We can use (7) to construct $\partial(\mathcal{G})$ from $\mathcal{G}$ inductively on the number of $\vee$-classes and show that if $\mathcal{G}$ is an RGB-cograph, then $\partial(\mathcal{G})$ is an RB-cograph. More precisely, if $\mathcal{G}$ is æ-connected and æ-acyclic then $\partial(\mathcal{G})$ and each of the $\partial\left(B_{1} \gamma \ldots \gamma B_{n}\right)$ determined by the $\vee$-classes of modalities are. For this, observe that, a priori, moving a $B_{i}$ out from the context could create or destroy æ-paths. However, we only claim that $æ-$ connectedness and æ-acyclicity are preserved, i.e., if the original RGB-cograph is correct, then so is the one constructed via Equation (7). By way of contradiction, assume the RB-cograph in the right-hand side sequent of Equation (7) contains a chordless æ-cycle and $\partial\left(B_{1}>2 \ldots B_{n}\right)$ is æ-connected. This chordless cycle cannot contain atoms from both $\bar{v}_{1}^{\prime} \otimes \cdots \otimes \bar{v}_{n}^{\prime} \otimes \operatorname{fm}\left(B_{1} \not \gamma \ldots \gamma B_{n}\right)$ and $\operatorname{fm}\left(F\left\{v_{1}^{\prime}\right\} \cdots\left\{v_{n}^{\prime}\right\}\right)$. If the cycle contains two vertices in $\mathrm{fm}\left(B_{1} \gamma \ldots \gamma B_{n}\right)$, then it must have chords, since $\partial\left(B_{1} \gamma \ldots \gamma B_{n}\right)$ is connected. Hence, the cycle cannot contain any $v_{i}^{\prime}$ or $\bar{v}_{i}^{\prime}$. This means that the cycle is fully contained inside the context $F\{\} \cdots\{\}$ or inside $B_{1} \gamma \ldots \gamma 8 B_{n}$. Therefore the cycle must already be present in the original RGB-cograph. Contradiction. Now pick any two vertices $x^{\prime}$ and $y^{\prime}$ in the right-hand side sequent of Equation (7). We show that there is a chordless æ-path between them. Let $x$ and $y$ be the corresponding vertices in the original RGB-cograph (if $x^{\prime}$ or $y^{\prime}$ are one of the $v_{i}^{\prime}$ or $\bar{v}_{i}^{\prime}$, take the corresponding $v_{i}$ ). By assumption there is a chordless æ-path between $x$ and $y$. We can recover this path in the right-hand side sequent of Equation (7). If the original path passes through a $v_{i}$, then in the new graph we can pass through the new edge $v_{i}^{\prime}-\bar{v}_{i}^{\prime}$. The converse is proved similarly. Figure 12 shows two examples of the definition of $\partial(\mathcal{G})$.

Sequentialization of $\mathcal{G}$ using $\partial(\mathcal{G})$. We can now piggyback on Retorés proof [44] of sequentialization for RB-cographs, to produce an MLL-K sequent proof for $\mathrm{fm}(\mathcal{G})$. Since $\partial(\mathcal{G})$ and each of the $\partial\left(B_{1} \gamma \ldots \gamma B_{n}\right)$ determined by the $\vee$-classes of modalities are $æ$-connected and æ-acyclic RB-cograph,

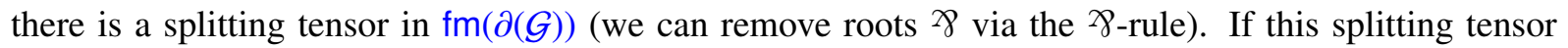
is also present in $\operatorname{fm}(\mathcal{G})$, we can directly apply the $\otimes$ rule and proceed by induction hypothesis. If it is not present in $\mathrm{fm}(\mathcal{G})$ then it must be of shape $\bar{v}_{1}^{\prime} \otimes \cdots \otimes \bar{v}_{n}^{\prime} \otimes \partial\left(B_{1} \not 8 \ldots \gamma B_{n}\right)$ and be introduced by the translation in Equation (7). Since $\partial(\mathcal{G})$ is æ-connected, we can without loss of generality assume the the context consists only of $v_{1}^{\prime}, \ldots, v_{n}^{\prime}$. Otherwise our tensor would not be splitting. Hence, we have

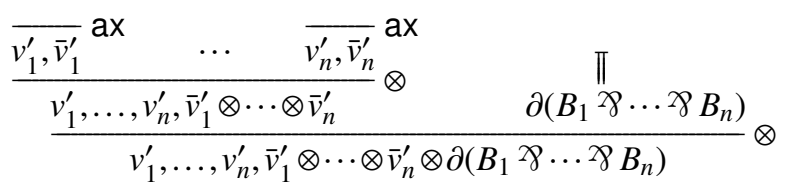

whose conclusion is $\partial\left(\left(v_{1} \triangleleft B_{1}\right) \not \gamma \ldots \gamma\left(v_{n} \triangleleft B_{n}\right)\right)$. Thus, we can apply the $\mathrm{w}$ ! $p$-rule and we can proceed by induction hypothesis.

Moreover, if $V_{\mathcal{G}}^{\circ} \neq \emptyset$, then we conclude similarly to the case of $\mathrm{MLL}_{\mathrm{u}}$-correct RGB-cographs.

We summarize the main results of this section by means of the following theorem:

Theorem 17. Let $\mathcal{G}$ be a RGB-cograph with $\lfloor\mathcal{G}\rfloor=\llbracket F \rrbracket$ and $\mathrm{X} \in\left\{\mathrm{MLL}, \mathrm{MLL}_{\mathrm{u}}, \mathrm{MELL}\right\}$. Then

$$
\text { F provable in } \mathrm{X}^{\ell} \Longleftrightarrow \mathcal{G} \text { is } \mathrm{X} \text {-correct }
$$



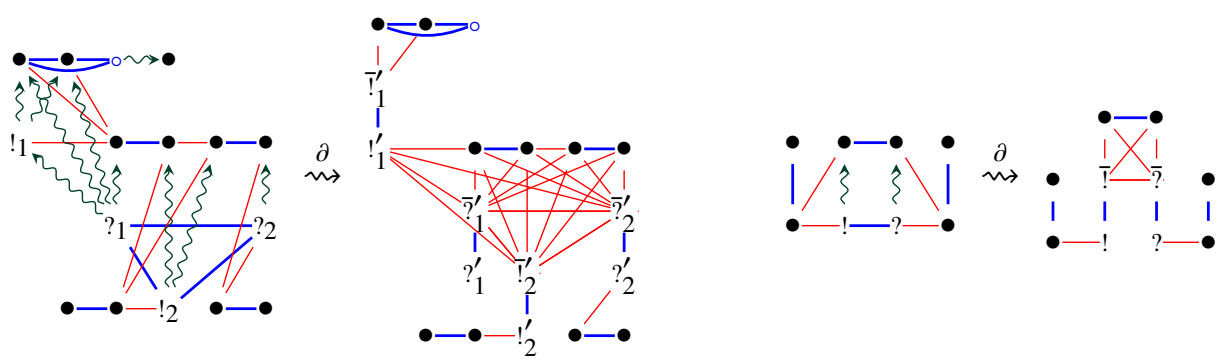

Figure 12: The RGB-cographs for $F_{1}=\bar{d} \not 8(d \otimes !(\bar{b} \otimes c) \not 8 \bar{e} \not 8(e \otimes ? \bar{c}) \not \gamma ?(b \otimes !(a \ngtr \bar{a}))) \not 8 ? f$ and $F_{2}=$ $b \ngtr(\bar{b} \otimes ! a) \not \supset(? \bar{a} \otimes c) \not 8 \bar{c}$, and the corresponding RB-cographs $\partial\left(F_{1}\right)$ and $\partial\left(F_{2}\right)$.

\section{MELL-Fibrations}

In this section we show how specific morphisms between modal relation webs, which we call MELLfibrations, allow us encode MELL $\downarrow$ derivations. We here present some of the results in [6] where skew fibrations are meant to capture (deep) weakening-contraction derivations in modal logic. In fact, thanks to additional definitions, we are able to restrain the these rules applications only to the ones on ?-formulas (or $\perp$ ). In the syntax proposed in this paper (deep) weakening-rules cannot properly be pushed down in the derivation since the information of the jump, represented by the propositional constant $\circ$, is firmly attached to an an axiom- or a 1-rule. Nevertheless, we separate the instantiation of a weakening (the jump appearing in a $\mathrm{ax}_{j}$ - or $1_{j}$ ) from the weakening application (the $\mathrm{w}_{?}^{\downarrow}$ - or $\perp^{\mathrm{j} \downarrow}$-rule).

Definition 18. Let $\mathcal{G}$ and $\mathcal{H}$ be modal relation webs . A linear fibration $f: \mathcal{G} \rightarrow \mathcal{H}$ is a function from $V_{\mathcal{G}}$ to $V_{\mathcal{H}}$ such that

1. $f$ preserves $\frown$ and $\rightsquigarrow$, that is, if $v R_{\mathcal{G}} w$ then $f(v) R_{\mathcal{H}} f(w)$ for $R \in\{\frown, \rightsquigarrow\}$ :

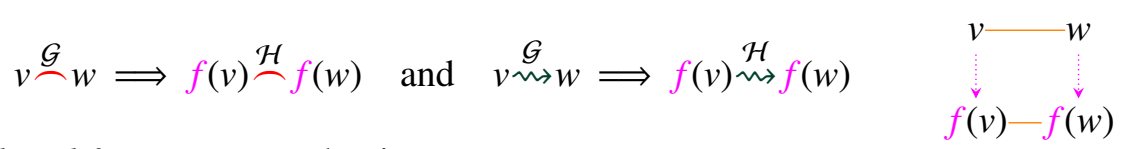

2. $f$ has the skew-lifting property, that is

$$
\begin{aligned}
& \text { for every } v \in V_{\mathcal{G}} \text { and } w \in V_{\mathcal{H}} \text { and } R \in\{\frown, \rightsquigarrow\} \text { with } w R_{\mathcal{H}} f(v), \\
& \text { there is a } u \in V_{\mathcal{G}} \text { such that } u R_{\mathcal{G}} v \text { and } w \leftarrow f(u) \text { and } w \not \mathcal{H} \\
& \mathcal{H}
\end{aligned}
$$

3. $f$ is modal, that is

$$
\begin{aligned}
& \text { if } u \stackrel{\mathcal{G}}{\mathcal{G}} v \text { and } f(u) \stackrel{\mathcal{H}}{m} f(v) \text {, then there is a } w \in V_{\mathcal{G}} \text { such that } \\
& w \stackrel{\mathcal{G}}{w \rightarrow v} \text { and } f(u)=f(w) \text {, or } u \stackrel{\leftrightarrow}{\rightsquigarrow} \text { and } f(v)=f(w) .
\end{aligned}
$$

4. $f$ preserves non-jump labels and assign jumps, that is

$$
\text { if } l(v) \neq \circ \text {, then } l(v)=l(f(v)) ; \quad \text { if } l(v)=\circ \text {, then } l(f(v)) \in\{\circ, \perp, ?\} .
$$

5. $f$ has the o-domination property, i.e.,

$$
\begin{aligned}
& \text { if } w \in V_{\mathcal{H}} \backslash f\left(V_{\mathcal{G}}\right) \text {, then there is a } u \in V_{\mathcal{G}}^{\circ} \text { such that } f(u) \rightsquigarrow w, l(f(u))=\text { ?, } \\
& \text { and if } R \in\{\frown, \rightsquigarrow\} \text {, then } f(v) R_{\mathcal{H}} f(u) \text { iff } f(v) R_{\mathcal{H}} w .
\end{aligned}
$$




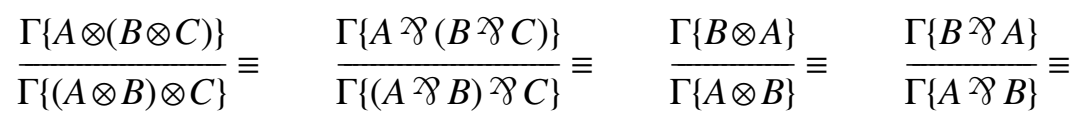

Figure 13: Deep rules for formula equivalence.

6. $f$ has the ?-domination property, i.e.:

$$
\begin{aligned}
\text { if } f\left(v_{1}\right)= & f\left(v_{2}\right) \text {, then there are } w_{1} \neq w_{2} \text { such that } f\left(w_{1}\right)=f\left(w_{2}\right)=w, l(w)=? \text { and } \\
& \text { either } w_{1}=v_{1} \text { and } w_{2}=v_{2} \quad \text { or } w_{1} \rightsquigarrow v_{1} \text { and } w_{2} \rightsquigarrow v_{2}
\end{aligned}
$$

The conditions in Theorem 18 have a simple interpretation if we identify the vertices of $\mathcal{G}$ and $\mathcal{H}$ with the atoms, $\perp, \circ$, ? and ! occurring in the corresponding encoded formulas $G$ and $H$, and the and nodes in the formula trees of $G$ and $H$. In fact, there is a $\frown$ between two vertices iff their least common ancestor in the formula tree is a $\otimes$. Similarly, there is a $\rightsquigarrow$ from a vertex $v$ to a vertex $w$ iff $w$ is an atom in the scope of a modality $v$; more precisely, $v$ is the least common ancestor of $v$ and $w$. According with this remark the above conditions have the following interpretation:

1. a linear fibration does not modify the least common ancestor of the corresponding nodes in the formula tree;

2. if the image of a vertex $u$ is a modality dominating the image of a vertex $v$, then there is a vertex $w$ with the same image of $u$ such that it is a modality dominating $v$;

3. a skew fibration can replace an internal node $n$ by a disjunction node with one child $n$ and the other child any formula tree; a skew fibration can attach a formula tree below a node of a modality with no child;

4. all labels except the $\circ$ are preserved by $f$. If $l(v)$, then $l(f(v))$ may be preserved or become either a $\perp$ or a ?;

5. if a vertex in $\mathcal{H}$ is not image of a vertex in $\mathcal{G}$, then there is a target of a $\rightsquigarrow$ with source a vertex $f(u)$ with such that $l(f(u))=$ ? and $l(u)=\circ$;

6. if a vertex $v$ of $\mathcal{H}$ is the image of $n>1$ distinct vertices $v_{1}, \ldots, v_{n}$ in $\mathcal{G}$, then each of these vertices is an atom or a modality of a formula of the shape ?A.

These conditions allow us to restraint the correspondence between contractions-weakening derivations and skew fibrations [23, 46, 6, 5, 34] on the formulas on the form ?A. However, since in relation web we consider formulas modulo associativity and commutativity of $\otimes$ and $>8$ (see Theorem 11 , we need to also consider the additional (deep) rules in Figure 13 taking care of this equivalence.

Proposition 19. If $\Gamma$ and $\Gamma^{\prime}$ are sequents, then $f: \llbracket \Gamma^{\prime} \rrbracket \rightarrow \llbracket \Gamma \rrbracket$ is a linear fibration iff $\Gamma^{\prime} \stackrel{\left\{w_{?}^{\downarrow}, \perp,,_{?}^{\downarrow}, c_{o}^{\downarrow} \equiv \downarrow\right\}}{\longmapsto} \Gamma$.

Proof. If $f$ and $f^{\prime}$ are linear fibrations, then by definition also $f \circ f^{\prime}$ is. We then conclude by showing that for any $\rho \in\left\{\mathrm{w}_{?}^{\downarrow}, \perp^{\downarrow}, \mathrm{c}_{?}^{\downarrow}, \mathrm{C}_{o}^{\downarrow}, \equiv \downarrow\right\}$ if $\rho \frac{F^{\prime}}{F}$, then there is a skew fibration $f_{\rho}: \llbracket F^{\prime} \rrbracket \rightarrow \llbracket F \rrbracket$.

If $\rho \in\{\equiv \downarrow\}$, then $f_{\rho}$ is an identity, hence a linear fibration. If $\rho \in\left\{\perp^{\downarrow}\right\}$, then $f_{\rho}$ is an identity preserving labels with the exception of a unique $u$ such that $l(u)=\circ$ and $l(f(u))=\perp$; thus $f_{\rho}$ is a linear fibration. If $\rho \in\left\{\mathbf{w}_{?}^{\downarrow}\right\}$, then $f_{\rho}$ is an identity over the image of $f_{\rho}$ preserving labels with the exception of a unique $u \in V^{\circ}$ such that $l(f(u))=$ ?. Moreover, any vertex $w$ in $\llbracket F \rrbracket$ which is not image of a vertex in $\llbracket F^{\prime} \rrbracket$ is 
dominated by $f(u)$, that is $f(v) \leadsto w$. If $\rho \in\left\{c_{?}^{\downarrow}\right\}$ then $F^{\prime}=\Gamma\{? A \ngtr ?$ ? $\}$ and $F=\Gamma\{? A\}, f_{\rho}$ restricted to $V_{\llbracket \Gamma\{\}]}$ is an identity. Moreover, $f_{\rho}$ preserves $\frown$ and $\rightsquigarrow$, is modal and has the ?-domination property (o-domination is trivially satisfied). The case if $\rho \in\left\{\mathbf{C}_{\circ}^{\downarrow}\right\}$ is proven similarly.

Conversely, by the result in [6, 34], we know that since $f$ is a modal skew fibration, then $\Gamma^{\prime} \mathrm{C}^{\downarrow}, \mathrm{W}^{\downarrow}, \equiv^{\downarrow} \Gamma^{\downarrow}$ where $\mathrm{W}$ and $\mathrm{C}$ are the classical logic weakening and contraction rules. To conclude the proof it suffices to show that the Conditions $4 \sqrt{6}$ restrict the application of a $W^{\downarrow}$ inside the scope of a ? image of a jumpvertex (i.e. a $\mathrm{w}_{\text {? }}^{\downarrow}$ ), and an application of a $C^{\downarrow}$ on ?-formulas, that is, an application of a $c_{?}^{\downarrow}$. Equation $(13)$ ensures that if a $\mathbf{W}^{\downarrow}$ has been applied, then a vertex $v$ with $l(v)=\circ$ has been mapped in $f(v)$ with $l(f(v))=$ ? and the weakened formula $A$ is entirely in the scope of this ?, that is, for every vertex $w \in V_{\llbracket A \rrbracket}$ we have $f(v) \rightsquigarrow w$. If no $W^{\downarrow}$ has been applied, since $f$ preserves non-jump labels, then either $l(f(u))=\circ$, or $l(f(v))=\perp-$ in which case a $\perp^{\downarrow}$ has been applied. Moreover, by 6 if a $C^{\downarrow}$ has been applied then the contracted formula $C$ is of the shape ?A. Otherwise Condition 6 fails. In particular, if $C=\perp$ or $C=! A$, the condition on labels fails; while if $C=A>B$ or $C=A \otimes B$, this condition fails the condition of existence of a the vertices $w_{1}$ and $w_{2}$.

In order to capture der? and dig? rules application, it suffices to adapt the results from [6].

Definition 20. We say that two vertices $v$ and $w$ in a relation web $\mathcal{G}$ are clones if for all $u$ with $u \neq v$ and $u \neq w$ we have $u R v$ iff $u R w$ for all $R \in\{\frown, \rightsquigarrow, \leftrightarrow m, \smile\}$. If $v=w$ then they are trivially clones.

A ?-map is a mapping $f: \mathcal{G} \rightarrow \mathcal{H}$ where $\mathcal{G}$ and $\mathcal{H}$ are modal relation webs, such that the following conditions are fulfilled:

- if $v \neq w$ and $f(v)=f(w)$, then $v$ and $w$ are clones in $\mathcal{G}, \stackrel{\mathcal{G}}{\sim} w, l(f(v))=l(w) \in\{$ ?, ○ $\}$;

- if $f(v) \neq f(w)$ then $v R_{\mathcal{G}} w$ implies $f(v) R_{\mathcal{H}} f(w)$ for any $R \in\{\frown, \rightsquigarrow, \sim,, \smile\}$;

- if $v \in V_{\mathcal{H}}$ is not in the image of $f$ then $l(v)=$ ? and there is a $w \in V_{\mathcal{H}}$ with $v \rightsquigarrow w$.

Proposition 21. Let $\Gamma$ and $\Gamma^{\prime}$ be sequents. Then, $\Gamma^{\prime} \stackrel{\operatorname{der}_{?}^{\downarrow}, \operatorname{dig}_{?}^{\downarrow}, \operatorname{dig}_{\circ}^{\downarrow}}{\longmapsto} \Gamma$ iff there is a ?-map $f: \llbracket \Gamma^{\prime} \rrbracket \rightarrow \llbracket \Gamma \rrbracket$.

Proof. The follows the result in [6] for $\left\{4^{\downarrow}, t^{\downarrow}\right\}$-maps. It suffices to remark that the modalities ! and ? of MELL behave similarly to the modalities $\square$ and $\diamond$ of S4, and that $\circ$ can be also considered as a $\diamond$ with no subformulas in its scope.

We can now define fibrations capturing MELL ${ }^{\downarrow}$-derivations.

Definition 22. Let $f: \mathcal{G} \rightarrow \mathcal{H}$ be a map between modal relation web. We say that

- $f$ is an MELL-fibration if $f=f^{\prime \prime} \circ f^{\prime}$ for some $f^{\prime}: \mathcal{G} \rightarrow \mathcal{G}^{\prime}$ and $f^{\prime \prime}: \mathcal{G}^{\prime} \rightarrow \mathcal{H}$, where $f^{\prime}$ is a linear fibration and $f^{\prime \prime}$ is a ?-map;

- $f$ is a MLL $_{u}-$ fibration if $f$ is a MELL-fibration with $V_{\mathcal{H}}^{! ?}=\emptyset$;

- $f$ is a MLL-fibration if $f$ is a bijection and $V_{\mathcal{H}}^{\circ} \cup V_{\mathcal{H}}^{! ?}=\emptyset$.

Theorem 23. Let $\Gamma$ and $\Gamma^{\prime}$ be sequents, then $\Gamma^{\prime} \stackrel{\mathrm{MELL}}{\longleftarrow} \Gamma$ iff there is a MELL-fibration $f: \llbracket \Gamma^{\prime} \rrbracket \rightarrow \llbracket \Gamma \rrbracket$.

Proof. As consequence of Theorem 5 , we have that $\Gamma^{\prime} \stackrel{\text { MELL }}{\longmapsto} \Gamma$ iff there is a sequent $\Gamma^{\prime \prime}$ such that

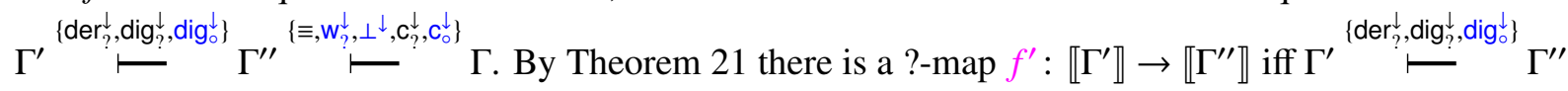




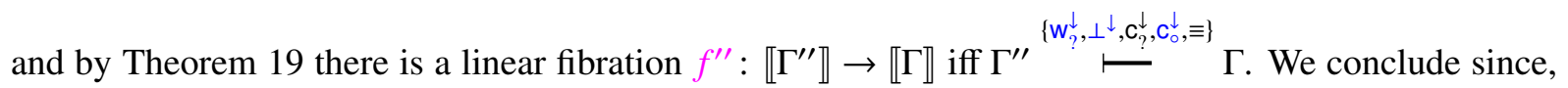
by definition, a MELL-fibration is the composition of a ?-map and a linear fibration.

To prove the converse, we define the relation web $\mathcal{H}^{\prime}$ such that $f=f^{\prime} \circ f^{\prime \prime}$ with $f^{\prime \prime}: \mathcal{H} \rightarrow \mathcal{H}^{\prime}$ and $f^{\prime}: \mathcal{H}^{\prime} \rightarrow \mathcal{G}$. We construct $\mathcal{H}^{\prime}$ from $\mathcal{H}$ as follows:

- for each pair of clones $v$ and $w$ with $v \rightsquigarrow w$ in $\mathcal{H}$ such that $f(v)=f(w)$, remove $v$;

- if $w \in V_{\mathcal{G}}$ such that $w \rightsquigarrow f(v)$ for some $v \in V_{\mathcal{H}}$ and $w=f(u)$ for no $u \in V_{\mathcal{H}}$, then add $w$ to $\mathcal{H}$ ad all needed edges such that $w R_{\mathcal{H}^{\prime}} v$ iff $w R_{\mathcal{G}} f(v)$ for $R \in\{\frown, \rightsquigarrow\}$.

We define $f^{\prime \prime}$ as the identity over non-clones vertices and as $f$ on clones vertices. We define $f^{\prime}$ in such a way $f=f^{\prime} \circ f^{\prime \prime}$. By definition $f^{\prime \prime}$ is a ?-map and $f^{\prime}$ is a linear fibration.

Proposition 24. If $\mathcal{H}$ and $\mathcal{G}$ are relation webs and $f: \mathcal{H} \rightarrow \mathcal{G}$ a function from $V_{\mathcal{H}}$ to $V_{\mathcal{G}}$, then it can be decided if $f$ is a MELL-fibration in time polynomial in $|\mathcal{G}|+|\mathcal{H}|$.

Proof. We decompose $f=f^{\prime} \circ f^{\prime \prime}$ using the same procedure used in the proof of Theorem 23 . To check if $f^{\prime \prime}$ is a ?-map we have to check if the label of all vertices which are not in the image of $f^{\prime \prime}$ are ?, and to check if the vertices with the same image are clones. This requires a quadratic time on the size of $V_{\mathcal{G}}$. Similarly, checking if $f^{\prime}$ is a linear fibration is quadratic on the size of $\mathcal{H}^{\prime}$, which is bounded by $\left|V_{\mathcal{H}}\right|+\left|V_{\mathcal{G}}\right|$.

\section{Combinatorial Proofs}

In this section we present a combinatorial proof syntax for $M E L L^{j}$ using the results in the previous sections. In particular, Theorem 5 gives us a decomposition result allowing us to separate in MELL ${ }^{j}$ derivation, the linear part, that is, MELL ${ }^{\ell}$, form the resource management part, that is, MELL $\downarrow$. The first part of the proof is encoded by RGB-cographs, while the second by MELL-fibrations.

Definition 25. A map $f: \mathcal{G} \rightarrow \llbracket F \rrbracket$ from an RGB-cograph $\mathcal{G}$ to a the relation web $\llbracket F \rrbracket$ is allegiant if the following conditions are satisfied:

- if $v, w \in V_{\mathcal{G}}^{\bullet}$ and $v \stackrel{\mathcal{G}}{v} w$ then $f(v)$ and $f(w)$ are labeled by dual atoms in $\mathcal{A}$;

- if $v \in V_{\mathcal{G}} \backslash V_{\mathcal{G}}^{\circ}$ then $l(f(v))=l(v)$;

- if $v \in V_{\mathcal{G}}^{\circ}$ then $l(f(v)) \in\{\perp, ?\}$.

Definition 26. For $\mathrm{X} \in\left\{\mathrm{MLL}, \mathrm{MLL}_{\mathrm{u}}, \mathrm{MELL}\right\}$, an $\mathrm{X}$-combinatorial proof of a sequent $\Gamma$ is an $\mathrm{X}$-fibration $f: \mathcal{G} \rightarrow \llbracket \Gamma \rrbracket$ from an X-correct RGB-cograph $\mathcal{G}$ to the relation web of $\Gamma$.

Theorem 27. If $F$ is a formula and $\mathrm{X} \in\left\{\mathrm{MLL}, \mathrm{MLL}_{\mathrm{u}}, \mathrm{MELL}\right\}$, then $\stackrel{\mathrm{X}}{\mathrm{F}}$ iff there is a $\mathrm{X}$-combinatorial proof $f: \mathcal{G} \rightarrow \llbracket F \rrbracket$.

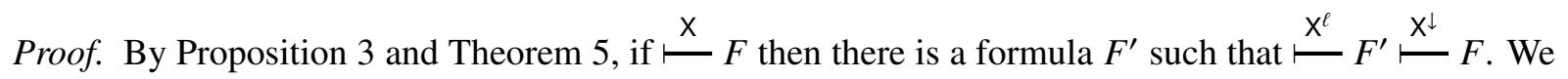
conclude by Theorems 17 and 23 .

After the result in [6] we have that MELL-combinatorial proofs represent two $\simeq$ J-equivalent proofs with the same syntactic object.

Proposition 28. Let $\pi_{1}$ and $\pi_{2}$ be two derivations in MELL' ${ }^{j}$, then $\pi_{1} \simeq \mathrm{J} \pi_{2}$ iff they are represented by the same MELL-combinatorial proof. 
Proof. It follows by the fact that MELL ${ }^{j}$ can be seen as modal logic S4 with additional axiom-like rules $\left(\mathrm{ax}_{j}\right.$ and $\left.1_{j}\right)$ and restricted versions of weakening and contraction rule. Note that, in [6] the proof equivalence is stated in such a way rule that permutations of $\mathrm{W}$ and $\mathrm{C}$ below $\mathrm{w} ! p$ are not allowed.

In particular, there is a one-to-one correspondence between $M L L_{u}$-combinatorial proofs and $M L L_{u}$ proof nets with jumps in [22].

Theorem 29. For all $\mathrm{X} \in\left\{\mathrm{MLL}, \mathrm{MLL}_{\mathrm{u}}, \mathrm{MELL}\right\}, \mathrm{X}$-combinatorial proofs are a sound and complete proof system for $\mathrm{X}$ in the sense of [9].

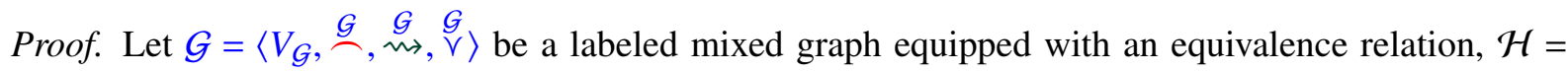
$\left\langle V_{\mathcal{H}}, \stackrel{\mathcal{H}}{\sim}, \stackrel{\mathcal{H}}{\rightsquigarrow}\right\rangle$ be a labeled mixed graph and $f: \mathcal{G} \rightarrow \mathcal{H}$ mapping vertices of $\mathcal{G}$ to vertices of $\mathcal{H}$. Checking if the two mixed graphs are relation web is polynomial by Theorem 10 on the sizes of $\left|V_{\mathcal{G}}\right|+\left|V_{\mathcal{H}}\right|$ and checking if $\mathcal{G}$ is an X-correct RGB-cograph is polynomial on $\left|V_{\mathcal{G}}\right|$. By Theorem 24 we have that checking if $f$ is a $\mathbf{X}$-fibration is polynomial on $\left|V_{\mathcal{G}}\right|+\left|V_{\mathcal{H}}\right|$. Then, since checking if $f$ is allegiant is linear on $\left|V_{\mathcal{G}}\right|+\left|V_{\mathcal{H}}\right|$, we conclude the proof.

\section{Handsome Proof Nets for MELL}

The combinatorial proofs defined in the previous section can be interpreted as an extension of both Retoré's [44] and Hughes' [23] syntaxes. They only allow to represent cut-free proofs as unfolded Retoré's proof nets [40] do. In this section we define exponentially handsome proof nets (or MELLcombinatorial proofs with cuts) by extending MELL-combinatorial proofs. We conclude by providing a cut-elimination procedure for exponentially handsome proof nets.

We adapt the solution proposed in [23, 24] where instances of cut are replaced by conjunction rules producing contradictions, i.e. formulas of the form $A \otimes \bar{A}$, which are later "discarded" at the end of the derivation. In the classical setting, this substitution creates no further interaction of the contradiction with any other rule in the derivation. The presence of the weak promotion rule prevents us from ignoring the presence of these contradictions, but we here propose a solution for this problem.

Remark 30. Another extension of combinatorial proof for classical logic allowing to represent proofs with cuts is given in [47] by means of combinatorial flows. In this setting cut is in some way encoded as sequential composition of flows. This syntax is strongly related with the property of the deep inference system SKS for classical logic [19] which states that if $\stackrel{\text { SKS }}{=} \bar{A}>B$, then $A \stackrel{\text { SKS }}{\longleftarrow} B$. Such a result is out of the scope of this paper, but it could be obtained by defining a deep inference system for MELL ${ }^{j}$ based on the systems in [45, 48].

Definition 31. If $F$ is a formula, we write $?^{n} F$ to denote the formula $?^{n} F=\overbrace{? \cdots ?}^{n \text { times }} F$. In particular, $?^{0} F=F$. A contradiction is a formula of the form $C=A \otimes \bar{A}$ for a MELL-formula $A$, and a weakencontradiction is a formula of the form $?^{n} C$ with $C$ a contradiction.

Theorem 32. Let $\Gamma$ be a sequent and $C_{1}, \ldots, C_{n}$ weaken-contradictions. If $\stackrel{\mathrm{MELL}}{\longleftarrow} \Gamma, C_{1}, \ldots, C_{n}$ then $\stackrel{\mathrm{MELL}}{\longleftarrow} \Gamma$. Conversely, if $\Gamma$ admits a derivation in MELL $\cup\{$ cut $\}$ containing $k$ occurrences of the cut-rule with pairs of active formulas $\left(A_{1}, \bar{A}_{1}\right), \ldots,\left(A_{k}, \bar{A}_{k}\right)$ then $\stackrel{\mathrm{MELL}}{\longleftarrow} \Gamma, ?^{n_{1}}\left(A_{1} \otimes \bar{A}_{1}\right), \ldots, ?^{n_{k}}\left(A_{i} \otimes \bar{A}_{i}\right)$ for some $n_{1}, \ldots, n_{k} \in \mathbb{N}$.

Proof. By induction on the number of weaken-contradictions formulas $k$. If $\Gamma, C$ is provable in MELL and $C=?^{n}(A \otimes \bar{A})$, then in any derivation of $\Gamma, C$ there is an occurrence of a rule $\otimes$ with principal formula 
$A \otimes \bar{A}$ and active formulas $A$ and $\bar{A}$. We can obtain a derivation of $\Gamma$ by replacing such an occurrence with a cut having the same active formulas, and then removing each occurrence of the active formula $A \otimes \bar{A}$ from the derivation.

$$
\frac{\Gamma, A \quad \bar{A}, \Delta}{\Gamma, A \otimes \bar{A}, \Delta} \otimes \quad \leadsto \quad \frac{\Gamma, A \quad \bar{A}, \Delta}{\Gamma, \Delta} \text { cut }
$$

Then we conclude thanks to Theorem 1 .

The converse is proven by similarly. In this case $n_{i}$ is defined as the number of occurrences of $\mathrm{w} ! p$ below the cut with active formulas formula $A_{i}$ and $\bar{A}_{i}$ for each $i \in\{1, \ldots k\}$.

Definition 33. An exponentially handsome proof net of a sequent $\Gamma$ is a MELL-combinatorial proof $f: \mathcal{G} \rightarrow \llbracket \Gamma, \Delta \rrbracket$ where $\Delta$ is a (possibly empty) sequent of weaken-contradictions.

In drawing such objects, we shade in gray the backgrounds of the portion of the relation web of $\Gamma$ containing the vertices of the weaken-contradictions (for a graphical example refer to Figure 1).

We conclude this section by defining a graphical rewriting allowing us to produce a MELL-combinatorial proof from an exponentially handsome proof net.

Theorem 34. If $f: \mathcal{G} \rightarrow \llbracket \Gamma, \Delta \rrbracket$ is an exponentially handsome proof nets of $\Gamma$, then there is a MELLcombinatorial proof $f^{\prime}: \mathcal{G} \rightarrow \llbracket \Gamma \rrbracket$.

Proof. If $\Delta=\emptyset$, then $f$ is a MELL-combinatorial proof. Otherwise, in order prove this result we implement cut-elimination by translating the rewriting in Figure 8 . We consider the following key cases which are shown in Figure 14

- $\underset{\sim \rightarrow c u t}{\operatorname{ax}-\mathrm{VS} \text {-ax }}$ : remove the two vertices in the contradiction and merge their $\vee$-classes;

$\perp$-VS-1

- ${ }_{n \rightarrow \text { cut }}$ : similarly to the previous one;

- $\stackrel{\otimes-\mathrm{VS}-{ }^{2} \mathrm{~g}}{\sim}$ : remove the $\frown$-edges between the vertices in $B$ and the ones in $A$ and $\bar{A}$, and remove the $\frown$-edges between the vertices in $A$ and the ones in $\bar{B}$;

- $\stackrel{\mathrm{w} ! p \text {-VS-w! } p}{\sim \text { cut }}$ : replace the $\frown$-edges between ? and a vertex in $A$ with $\rightsquigarrow$-edge from ? to a vertex in $A$. Merge the $\vee$-classes of ! and ? and then remove the vertex !;

- $\stackrel{\mathrm{w} ! p \text {-VS-der? }}{\sim}$ : remove the ! and the vertices in its $\vee$-class;

- $\stackrel{\mathrm{w} ! p \text {-VS-wj }}{\sim \text { cut }}:$ remove the ! and any vertex pointed by the ? in its $\vee$-class. Then replace each ? in with a new $\circ$ having the same image by $f$. Add to the $\vee$-class of the ${ }^{\text {cut }}$ which is in the pre-image of the ? of the cut-formula the new o-vertices. Finally remove ${ }^{\text {cut }}$.

- $\stackrel{\mathrm{w} ! p \text {-VS-c }}{\leadsto \text { cut }}:$ remove any $\frown$ connecting $! A$ to any copy of $? \bar{A}$; for each pre-mage of ? excepting one, make a copy of the connected component of the RGB-cograph containing the $\checkmark$-class of ! for each ? in the pre-image of the ? of the cut-formula ? $\bar{A}$. add the $\frown$-edges between each copy of !A and the corresponding copy of ? $\bar{A}$;

$\stackrel{\mathrm{w} ! p \text {-VS-dig? }}{\sim \rightarrow \text { cut }}$ : remove any $\frown$ connecting !A to any ? with image the ? of the cut-formula; make a copy of the $\vee$-class of ! for each ? in the pre-image of the ? of the cut-formula ? $\bar{A}$. Add the $\frown$-edge and the $\rightsquigarrow$-edges in such a way each new copy of ? and ! is a clone of the original one. Add a between each new copy of ! and the corresponding ?. 
Note that proof equivalence and the permutations in Figure 9 are already captured by the syntax.

Let $\Delta=C_{1}, \ldots, C_{n}$. We define the function $\mathrm{W}_{\Delta}$ associating to an exponentially handsome proof nets $g: \mathcal{H} \rightarrow \llbracket \Sigma \rrbracket$ the string $\mathrm{W}_{\Delta}(g)=\left(|\Sigma|_{C_{1}}, \ldots,|\Sigma|_{C_{n}}\right)$ where $|\Sigma|_{C}$ is the number of occurrences of the the formula $C$ in $\Sigma$. The reduction strategy consists in selecting and applying reduction to one weakencontradiction in $f$ at a time, selecting the weaken-contradictions according with the following the order on $\Delta$ : we do not select a weaken-contradiction $C_{i+1}$ until we have not removed all the occurrences of $C_{i}$. At the end of each sub-routine removing an occurrence of a weaken-contradiction $C_{i}$, we may have created new copies of the weaken-contradictions, but only copies of the $C_{j}$ with $j>i$. The reduction order is the lexicographic order on the pair $\left(\left|V_{\llbracket C \rrbracket}\right|, \mathrm{W}_{\Delta}(g)\right)$ where $C$ is the currently selected weakencontradiction in the elimination process. Since each rewriting rule in Figure 14 either reduces $|\llbracket C \rrbracket|$ or $\mathrm{W}_{\Delta}$, then the cut-elimination procedure ends.

\section{Conclusions}

In this paper we extended Retoré's RB-cograph syntax for multiplicative proof nets [44] in order to include units and exponentials, refining the combinatorial proofs for modal logic from [6].

Aware of the limits in designing a syntax able to capture proof equivalence and with a polynomial correctness criterion [21], we restrained the notion of proof equivalence by introducing the proof system MELL ${ }^{j}$ for MELL, enforcing a coarser proof equivalence. We topologically characterized mixed graphs equipped with vertices partitions encoding linear proofs in MELL ${ }^{j}$, as well as the graph homomorphisms capturing the resource management part of proofs in MELL ${ }^{j}$. Using these results we introduced combinatorial proofs for MELL and then exponentially handsome proof nets for MELL, defined as compositions of combinatorial proofs. We also provided a normalization procedure by means of graph rewriting rules.

The RGB-cographs can be interpreted as a coherent interaction graphs [33] for MLL $\cup\{\mathrm{w} ! p\}$. We foresee a further application of exponentially handsome proof nets to explore the geometry of interaction of MELL [16] using the same approach from [14], where handsome proof nets are employed to investigate coherent spaces of MLL.

On Removing Jumps. Is it possible to modify exponentially handsome proof nets by removing jumps and thus recover a less coarse notion of proof equivalence including jump rewiring, at the price of loosing the polynomiality of the correctness criterion.

For this purpose, we should consider the following set of rules:

$$
\left\{a x, 1, \circ, 28, \otimes, \operatorname{mix}, \mathrm{w} ! p, \operatorname{der}_{?}, \operatorname{dig}_{?}, \operatorname{dig}_{\circ}, \perp^{j}, \mathrm{w}^{j}, \mathrm{c}_{?}\right\} \quad \text { with } \quad \frac{\Gamma \Delta}{\Gamma, \Delta} \operatorname{mix} \quad \text { and } \quad \mathrm{o}_{\circ}^{\circ}
$$

A correctness criterion for RGB-cographs encoding linear proofs of this proof system, that is, derivations

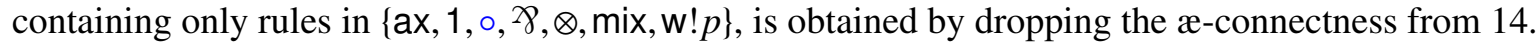

Note that we cannot get rid of $\circ$ in presence of the restricted weakening rule of linear logic: without placeholders we could not represent the proof below on the left, because the skew lifting condition (see Equation (10) would fail for any $\frown$-edge connecting a vertex in $A$ with a vertex in $B$.

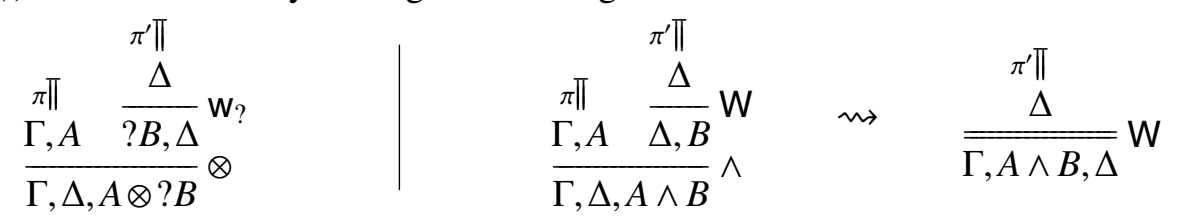

The use of excising, that is, the transformation above on the right cannot be used to overcome this 


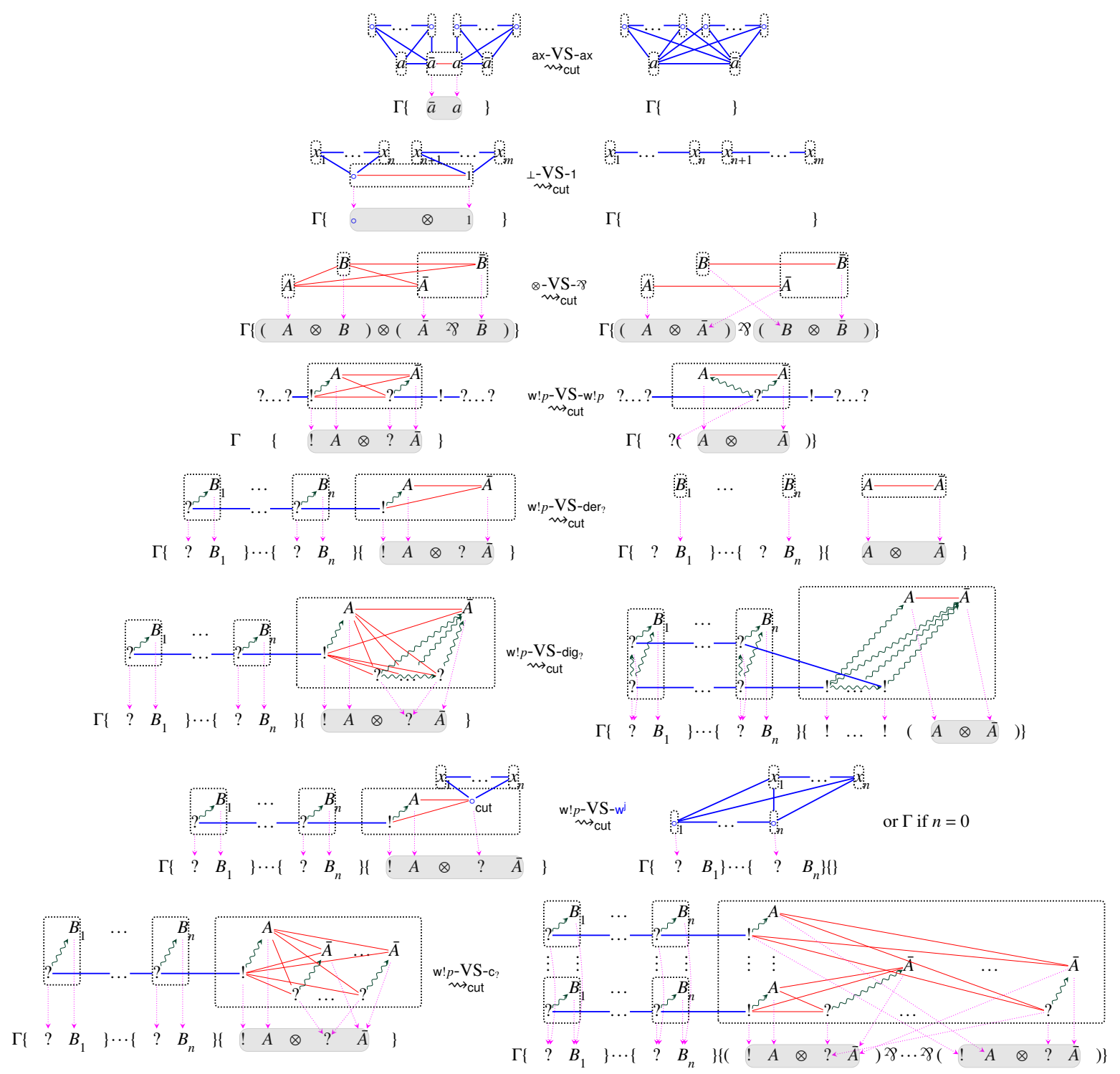

Figure 14: Cut-elimination steps for exponentially handsome proof nets. Dotted lines delimit modules, that is, sets of vertices having the same $\frown$ - and $\rightsquigarrow$-relation with any vertex outside. To simplify the reading we write with the same symbol a formula and its pre-image by $f$.

problem as it is done in classical logic (see [23, 4]) since, in linear logic, this proof transformation cannot be performed.

On Generalized ?-Nodes. The rule permutations allowing to push weakening and contraction below the promotion rule (see last line of Figure 5) are allowed in MELL proof nets, thanks to the so-called generalized ?-nodes [10]. The syntax with generalized ?-nodes captures these equivalences, allowing a "flexible" representation of boxes, which are the syntactic elements representing promotion rules. In fact, generalized ?-nodes allow to identify nets where boxes may have different amounts of auxiliary ports. This depends on the fact that boxes have a different status with respect to the interaction net syntax [27].

In exponentially handsome proof nets, each (weak) promotion is rigidly encoded: each !-vertex is the 
principal port of a box and each auxiliary port is encoded by a ?-vertex in the same $\checkmark$-class. In particular, this allows us to keep track of the depth of a propositional letter $a$, a $\circ$ or a 1 in terms of the number of incoming $\rightsquigarrow$ in the RGB-cograph. This clearly prevents any rule permutation which may change the number of auxiliary ports of a box.

\section{Acknowledgements}

I wish to thank the anonymous reviewers, who gave very valuable feedback and pointed me to relevant literature. I also would like to thank Christian Retoré, Lutz Straßburger, Giti Omidvar for the useful feedbacks and technical discussions.

\section{References}

[1] Beniamino Accattoli (2013): Linear Logic and Strong Normalization. In Femke van Raamsdonk, editor: 24th International Conference on Rewriting Techniques and Applications (RTA 2013), Leibniz International Proceedings in Informatics (LIPIcs) 21, Schloss Dagstuhl-Leibniz-Zentrum fuer Informatik, Dagstuhl, Germany, pp. 39-54, doi:10.4230/LIPIcs.RTA.2013.39

[2] Matteo Acclavio (2016): String diagram rewriting: applications in category and proof theory. $\mathrm{Ph} . \mathrm{D}$. thesis, Aix-Marseille Université. Available at matteoacclavio.com/Archive/PhdThesisAcclavio.pdf.

[3] Matteo Acclavio (2019): Proof diagrams for multiplicative linear logic: Syntax and semantics. Journal of Automated Reasoning 63(4), pp. 911-939, doi:10.1007/s10817-018-9466-4.

[4] Matteo Acclavio \& Lutz Straßburger (2018): From Syntactic Proofs to Combinatorial Proofs. In Didier Galmiche, Stephan Schulz \& Roberto Sebastiani, editors: Automated Reasoning, Springer International Publishing, Cham, pp. 481-497, doi:10.1007/978-3-319-94205-6_32.

[5] Matteo Acclavio \& Lutz Straßburger (2019): On Combinatorial Proofs for Logics of Relevance and Entailment. In Rosalie Iemhoff, Michael Moortgat \& Ruy de Queiroz, editors: Logic, Language, Information, and Computation, Springer Berlin Heidelberg, Berlin, Heidelberg, pp. 1-16, doi:10.1007/978-3-662-59533-6_1.

[6] Matteo Acclavio \& Lutz Straßburger (2019): On Combinatorial Proofs for Modal Logic. In Serenella Cerrito \& Andrei Popescu, editors: Automated Reasoning with Analytic Tableaux and Related Methods, Springer International Publishing, Cham, pp. 223-240, doi:10.1007/978-3-030-29026-9_13.

[7] Denis Bechet, Philippe de Groote \& Christian Retoré (1997): A complete axiomatisation for the inclusion of series-parallel partial orders. In Hubert Comon, editor: Rewriting Techniques and Applications, Springer Berlin Heidelberg, Berlin, Heidelberg, pp. 230-240, doi:10.1007/3-540-62950-5_74.

[8] Kai Brünnler \& Alwen Fernanto Tiu (2001): A Local System for Classical Logic. In Robert Nieuwenhuis \& Andrei Voronkov, editors: Logic for Programming, Artificial Intelligence, and Reasoning, Springer Berlin Heidelberg, Berlin, Heidelberg, pp. 347-361, doi:10.1007/3-540-45653-8_24.

[9] Stephen A. Cook \& Robert A. Reckhow (1979): The relative efficiency of propositional proof systems. Journal of Symbolic Logic 44(1), p. 36-50, doi:10.2307/2273702.

[10] V. Danos \& L. Regnier (1995): Proof-Nets and the Hilbert Space. In: Proceedings of the Workshop on Advances in Linear Logic, Cambridge University Press, USA, p. 307-328, doi: $10.1017 /$ CBO9780511629150.016.

[11] Vincent Danos (1990): La logique linéaire appliquée à l'étude de divers processus de normalisation (principalement du $\lambda$-calcul). Thèse de Doctorat, Université Paris VII.

[12] Vincent Danos \& Laurent Regnier (1989): The structure of multiplicatives. Arch. Math. Log. 28(3), pp. 181-203, doi:10.1007/BF01622878

[13] R.J Duffin (1965): Topology of series-parallel networks. Journal of Mathematical Analysis and Applications 10(2), pp. 303-318, doi:10.1016/0022-247X(65)90125-3. 
[14] Thomas Ehrhard (2014): A New Correctness Criterion for MLL Proof Nets. In: Proceedings of the Joint Meeting of the Twenty-Third EACSL Annual Conference on Computer Science Logic (CSL) and the TwentyNinth Annual ACM/IEEE Symposium on Logic in Computer Science (LICS), CSL-LICS '14, Association for Computing Machinery, New York, NY, USA, pp. 1-10, doi:10.1145/2603088.2603125

[15] Jean-Yves Girard (1987): Linear logic. Theoretical Computer Science 50(1), pp. 1-101, doi:10.1016/03043975(87)90045-4.

[16] Jean-Yves Girard (1989): Towards a geometry of interaction. Contemporary Mathematics 92(69-108), p. 6, doi $10.1090 /$ conm/092/1003197.

[17] Jean-Yves Girard (1998): Light Linear Logic. Information and Computation 143(2), pp. 175-204, doi:10.1006/inco.1998.2700 Available at https://www.sciencedirect.com/science/article/pii/ S0890540198927006

[18] S. Guerrini (1999): Correctness of multiplicative proof nets is linear. In: Proceedings. 14th Symposium on Logic in Computer Science (Cat. No. PR00158), pp. 454-463, doi:10.1109/LICS.1999.782640.

[19] Alessio Guglielmi (2007): A System of Interaction and Structure. ACM Trans. Comput. Logic 8(1), p. 1-es, doi $10.1145 / 1182613.1182614$

[20] Alessio Guglielmi \& Lutz Straßburger (2001): Non-commutativity and MELL in the Calculus of Structures. In Laurent Fribourg, editor: Computer Science Logic, Springer Berlin Heidelberg, Berlin, Heidelberg, pp. 54-68, doi: $10.1007 / 3-540-44802-0 \_5$

[21] Willem Heijltjes \& Robin Houston (2014): No Proof Nets for MLL with Units: Proof Equivalence in MLL is PSPACE-Complete. In: Proceedings of the Joint Meeting of the Twenty-Third EACSL Annual Conference on Computer Science Logic (CSL) and the Twenty-Ninth Annual ACM/IEEE Symposium on Logic in Computer Science (LICS), CSL-LICS '14, Association for Computing Machinery, New York, NY, USA, pp. 50:150:10, doi $10.1145 / 2603088.2603126$.

[22] Dominic Hughes (2005): Simple Multiplicative Proof Nets with Units. Available at http://arxiv.org/ abs/math.CT/0507003 Preprint.

[23] Dominic J. D. Hughes (2006): Proofs without Syntax. Annals of Mathematics 164(3), pp. 1065-1076, doi $10.2307 / 20160016$

[24] Dominic J.D. Hughes (2006): Towards Hilbert's 24th Problem: Combinatorial Proof Invariants: (Preliminary version). Electronic Notes in Theoretical Computer Science 165, pp. 37-63, doi $10.1016 / j$.entcs.2006.05.036 Proceedings of the 13th Workshop on Logic, Language, Information and Computation (WoLLIC 2006).

[25] A.P Kopylov (2001): Decidability of Linear Affine Logic. Information and Computation 164(1), pp. 173-198, doi $10.1006 /$ inco.1999.2834

[26] Yves Lafont (1989): Interaction nets. In: Proceedings of the 17th ACM SIGPLAN-SIGACT symposium on Principles of programming languages, pp. 95-108.

[27] Yves Lafont (1995): From Proof Nets to Interaction Nets. In J.-Y. Girard, Y. Lafont \& L. Regnier, editors: Advances in Linear Logic, London Mathematical Society Lecture Notes 222, Cambridge University Press, pp. 225-247, doi:10.1017/CBO9780511629150.012.

[28] Yves Lafont (2004): Soft linear logic and polynomial time. Theoretical Computer Science 318(1), pp. 163180, doi $10.1016 / j . t c s .2003 .10 .018$. Implicit Computational Complexity.

[29] François Lamarche (1994): Proof nets for intuitionistic linear logic I: Essential nets. Technical Report, Imperial College, London.

[30] Olivier Laurent (2002): Étude de la polarisation en logique. Theses, Université de la Méditerranée - AixMarseille II. Available at https://tel . archives-ouvertes.fr/tel-00007884.

[31] Olivier Laurent (2020): Polynomial time in untyped elementary linear logic. Theoretical Computer Science 813, pp. 117-142, doi:10.1016/j.tcs.2019.10.002. 
[32] Damiano Mazza (2005): Multiport Interaction Nets and Concurrency. In Martín Abadi \& Luca de Alfaro, editors: CONCUR 2005 - Concurrency Theory, Springer Berlin Heidelberg, Berlin, Heidelberg, pp. 21-35, doi: $10.1007 / 11539452 \_6$

[33] Lê Thành Dũng Nguyen \& Thomas Seiller (2019): Coherent Interaction Graphs. In Thomas Ehrhard, Maribel Fernández, Valeria de Paiva \& Lorenzo Tortora de Falco, editors: Proceedings Joint International Workshop on Linearity \& Trends in Linear Logic and Applications, Oxford, UK, 7-8 July 2018, Electronic Proceedings in Theoretical Computer Science 292, Open Publishing Association, pp. 104-117, doi: $10.4204 /$ EPTCS.292.6.

[34] Benjamin Ralph \& Lutz Straßburger (2019): Towards a Combinatorial Proof Theory. In Serenella Cerrito \& Andrei Popescu, editors: Automated Reasoning with Analytic Tableaux and Related Methods, Springer International Publishing, Cham, pp. 259-276, doi:10.1007/978-3-030-29026-9_15.

[35] Laurent Regnier (1992): Lambda-calcul et réseaux. Ph.D. thesis, Paris 7.

[36] Christian Retoré (1987): Le système F en logique linéaire. Master's thesis, Université Paris 7, Paris.

[37] Christian Retoré (1993): Réseaux et séquents ordonnés. Theses, Université Paris-Diderot - Paris VII. Available at https://tel.archives-ouvertes.fr/tel-00585634

[38] Christian Retoré (1996): Calcul de Lambek et Logique Linéaire. Traitement Automatique des Langues 37(2), pp. 39-70.

[39] Christian Retoré (1996): Perfect matchings and series-parallel graphs: multiplicatives proof nets as REBgraphs. Electronic Notes in Theoretical Computer Science 3, doi:10.1016/S1571-0661(05)80416-5.

[40] Christian Retoré (1999): Handsome Proof-nets: REvB-Graphs, Perfect Matchings and Series-parallel Graphs. Research Report RR-3652, INRIA. Available at https://hal .inria.fr/inria-00073020.

[41] Christian Retoré (1999): Pomset logic as a calculus of directed cographs. In V. M. Abrusci \& C. Casadio, editors: Dynamic Perspectives in Logic and Linguistics: Proof Theoretical Dimensions of Communication Processes,Proceedings of the 4th Roma Workshop, Bulzoni, Roma, pp. 221-247. INRIA Research Report RR-3714 https://hal.inria.fr/inria-00072953

[42] Christian Retoré (1999): Pomset Logic as a Calculus of Directed Cographs. In V. M. Abrusci \& C. Casadio, editors: Dynamic Perspectives in Logic and Linguistics, Bulzoni, Roma, pp. 221-247. Also available as INRIA Rapport de Recherche RR-3714.

[43] Christian Retoré (2021): Pomset logic: The other approach to non commutativity in logic. In Claudia Casadio \& Philip J. Scott, editors: Joachim Lambek: on the interplay of mathematics, logic and linguistics, Outstanding contributions to logic, Springer Verlag, pp. 299-246, doi 10.1007/978-3-030-66545-6

[44] Christian Retoré (2003): Handsome proof-nets: perfect matchings and cographs. Theoretical Computer Science 294(3), pp. 473-488, doi 10.1016/S0304-3975(01)00175-X. Linear Logic.

[45] Lutz Straßburger (2003): Linear Logic and Noncommutativity in the Calculus of Structures. Ph.D. thesis, Technische Universität Dresden.

[46] Lutz Straßburger (2007): A Characterization of Medial as Rewriting Rule. In Franz Baader, editor: Term Rewriting and Applications, Springer Berlin Heidelberg, Berlin, Heidelberg, pp. 344-358, doi 10.1007/9783-540-73449-9_26.

[47] Lutz Straßburger (2017): Combinatorial Flows and Their Normalisation. In Dale Miller, editor: 2nd International Conference on Formal Structures for Computation and Deduction (FSCD 2017), Leibniz International Proceedings in Informatics (LIPIcs) 84, Schloss Dagstuhl-Leibniz-Zentrum fuer Informatik, Dagstuhl, Germany, pp. 31:1-31:17, doi 10.4230/LIPIcs.FSCD.2017.31.

[48] Lutz Straßburger (2003): MELL in the calculus of structures. Theoretical Computer Science 309(1), pp. 213-285, doi:10.1016/S0304-3975(03)00240-8.

[49] Lutz Straßburger (2019): On the decision problem for MELL. Theoretical Computer Science 768, pp. 91-98, doi $10.1016 / j . t c s .2019 .02 .022$. 
[50] Lorenzo Tortora de Falco (2000): Additives of linear logic and normalization-Part II: the additive standardization theorem.

[51] Lorenzo Tortora de Falco (2003): Additives of linear logic and normalization-Part I: a (restricted) ChurchRosser property. Theoretical Computer Science 294(3), pp. 489-524, doi:10.1016/S0304-3975(01)00176-1.

[52] Anne Sjerp Troelstra \& Helmut Schwichtenberg (2000): Basic proof theory, Second Edition. Cambridge tracts in theoretical computer science 43, Cambridge University Press, doi: 10.1017/CBO9781139168717. 